\title{
Metal-free, Regio-, and Stereo-Controlled Hydrochlorination and Hydrobromination of Ynones and Ynamides
}

\author{
Xiaojun Zeng, ${ }^{\dagger}$ Zhichao Lu, $^{\ddagger}$ Shiwen Liu, ${ }^{\dagger}$ Gerald B. Hammond, ${ }^{*}{ }^{\ddagger}$. \\ ${ }^{\dagger}$ College of Chemistry, Chemical Engineering and Biotechnology, Donghua University, 2999 North Renmin Lu, Shanghai 201620, \\ China \\ ${ }^{\ddagger}$ Department of Chemistry, University of Louisville, Louisville, Kentucky 40292, United States
}

Supporting Information

ABSTRACT: We developed an atom-economical and metalfree method for the regio- and stereo-selective hydrohalogenation of ynones and ynamides using easy to handle $\mathrm{DMPU} / \mathrm{HX}(\mathrm{X}=\mathrm{Br}$ or $\mathrm{Cl})$ reagents. The reaction operates under mild conditions and a range of functional groups is well tolerated. We propose that the hydrohalogenation of ynones gives the anti-addition products via a concerted multimolecular $\operatorname{Ad}_{\mathrm{E}} 3$ mechanism and that the hydrohalogenation of ynamides produces the syn-addition products via a cationic keteniminium intermediate.

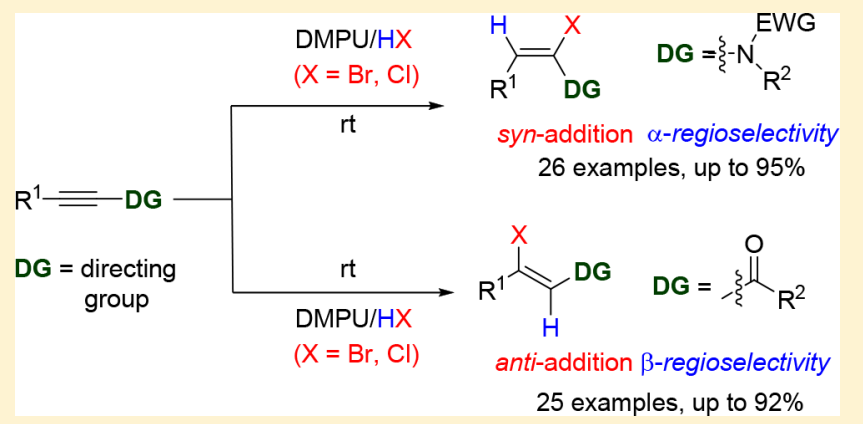

Scheme 1. Halogenation of Ynones and Ynamides

\section{INTRODUCTION}

Vinylhalides are important targets in medicine and materials, and they are also important substrates for cross-coupling reactions. ${ }^{1}$ More specifically, halogenated vinyl ketones and halogenated $\mathrm{N}$-vinyl amides (enamides) are especially important targets and synthetic building blocks because they are highly functional sources of vinyl halides. ${ }^{2}$ The hydrohalogenation of alkynes has provided a uniform approach toward viny halides, ${ }^{1 \mathrm{~g}, 3}$ and the most straightforward and atomeconomical synthesis of halogenated vinyl ketones and haloethenamides has been the regio- and stereo-selective hydrohalogenation of ynones and ynamides, respectively (Scheme 1, top). For example, the hydrohalogenation of ynones using $\mathrm{HBr} /$ water, ${ }^{4} \mathrm{AlBr}_{3} / \mathrm{H}_{2} \mathrm{O}$ or $\mathrm{LiBr} / \mathrm{TFA},{ }^{4 c} \mathrm{HCl}$ / water, ${ }^{5}$ and $\mathrm{SnCl}_{4} / \mathrm{H}_{2} \mathrm{O}^{5,6}$ (Scheme $1 \mathrm{a}-\mathrm{e}$ ) have been reported, but these methods give poor chemical yields due to side reactions, such as hydration. For the hydrohalogenation of ynamides, Hsung and co-workers reported a stereoselective synthesis of 1-haloenamides using in situ generated HX from $\mathrm{MgX}_{2}$ in a wet solvent (Scheme 1f). ${ }^{7}$ Iwasawa and co-workers reported the hydrochlorination of terminal ynamides using $\mathrm{HX}$ generated in situ from TMSX and water (Scheme $1 \mathrm{~g}$ ). ${ }^{8}$ Sahoo and co-workers demonstrated a one-pot construction of haloenamides using a $\mathrm{Ph}_{3} \mathrm{P} / \mathrm{H}_{2} \mathrm{O} / \mathrm{CX}_{4}$ system as the halide source (Scheme 1h). ${ }^{9}$ Recently, Shin and co-workers reported a novel benzoic acid-catalyzed hydrohalogenation of ynamides using $\mathrm{N}$-oxides/dihalogenated ethane system (Scheme $1 \mathrm{~d})^{10}$ whereas Thibaudeau and co-workers reported a syn-addition of $\mathrm{HF}$ to the ynamides. ${ }^{11}$ Although the above-mentioned examples underscore the importance of halogenation protocols, the reported methods are either too narrow in scope, or suffer from complex reaction conditions, low chemical yields, and/or

literature systems for
halogenation of ynones 1
a) $\mathrm{HBr} / \mathrm{H}_{2} \mathrm{O}$
c) $\mathrm{HX} / \mathrm{H}_{2} \mathrm{O}$
d) $\mathrm{SnCr}{ }_{3} / \mathrm{H}_{2} \mathrm{O}$
e) $\mathrm{LiBr} / \mathrm{TFA}$

$$
\begin{aligned}
& \text { literature systems for } \\
& \text { halogenation of ynamides } 4 \\
& \text { f) } \\
& \text { i) } \\
& \text { h) }
\end{aligned}
$$

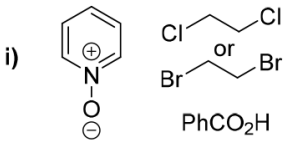

e) $\mathrm{LiBr} / \mathrm{TFA}$

1) low atom ecomomy; 2) poor yields (hydration by products etc.) 3) sometimes low steoro- selectivity

This work - an unified hydrohalogenation protocol

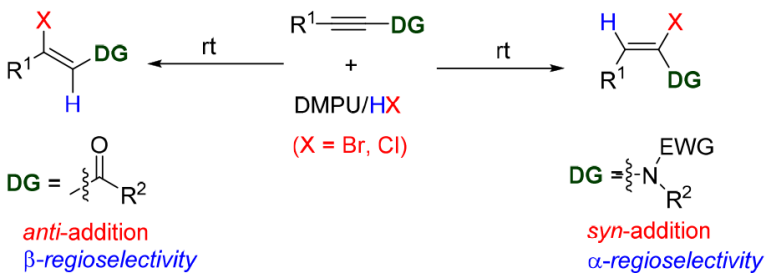

poor atom-economy. What is needed is a simple, unified, chemo- and stereo-selective atom-economical hydrohalogenation method for a wide range of ynones and ynamides.

Received: September 7, 2017

Published: November 23, 2017 
Recently, we reported a metal-free, hydrogen bonding cluster-enabled addition of sulfonic acids to haloalkynes. ${ }^{12}$ We now postulate that a metal-free and atom-economic hydrochlorination or hydrobromination of ynones $\mathbf{1}$ and ynamides $\mathbf{4}$ is possible using HX. However, hydrogen halides, such as hydrogen chloride and hydrogen bromide, are hazardous gases at room temperature. Although many $\mathrm{HX}$ reagents (e.g., $\mathrm{Et}_{2} \mathrm{O} / \mathrm{HCl}, \mathrm{H}_{2} \mathrm{O} / \mathrm{HCl}$, isopropanol/ $\mathrm{HCl}$, $\mathrm{HOAc} / \mathrm{HBr}, \mathrm{H}_{2} \mathrm{O} / \mathrm{HBr}$ ) are commercially available, they exist either in low concentration (e.g., $\mathrm{Et}_{2} \mathrm{O} / \mathrm{HCl}$ ) and/or in protic media $\left(\mathrm{H}_{2} \mathrm{O}\right.$, isopropanol, $\left.\mathrm{HOAc}\right)$ where the protic solvent hampers the nucleophilicity of $\mathrm{HX}$ and also competes with $\mathrm{HX}$ in nucleophilic addition. This effect may explain the low efficiency when protic solutions of HX are used (Scheme 1). In this regard, an HX solution in a neutral aprotic medium would be optimal to prevent competitive solvolysis reaction and to achieve high reactivity. Due to its high hydrogen bond basicity, yet low Brønsted basicity, ${ }^{13,14}$ DMPU (1,3-dimethyl-3,4,5,6tetrahydro-2 $(1 \mathrm{H})$-pyrimidinone) can form a stable and highly concentrated complex with hydrogen chloride $(\mathrm{HCl})$ and hydrogen bromide ( $\mathrm{HBr}$ ); we have used these complexes in nanogold catalyzed or noncatalyzed hydrohalogenation of alkenes and alkynes. ${ }^{15} \mathrm{We}$ are now glad to report an atomeconomical and metal-free method for the regio- and stereoselective hydrohalogenation of ynones and ynamides using easy to handle DMPU/HX ( $\mathrm{X}=\mathrm{Br}$ or $\mathrm{Cl})$ reagents.

\section{RESULTS AND DISCUSSION}

We carried out the hydrochlorination of 1a as our model reaction (Table 1). We were delighted to find that $\mathrm{DMPU} / \mathrm{HCl}$

Table 1. Optimization of the Hydrochlorination of Alkynyl Ketone $^{a}$

solvent

\begin{tabular}{cllc} 
entry & \multicolumn{1}{c}{$\mathrm{HCl}$ reagent } & solvent & $\mathbf{2} /{ }^{b}$ (2a:2a') (anti:syn) \\
1 & $\mathrm{Et}_{2} \mathrm{O} / \mathrm{HCl}$ & $\mathrm{DCM}$ & $70(6: 1)$ \\
2 & 1,4-dioxane $/ \mathrm{HCl}$ & $\mathrm{DCM}$ & $81(7: 1)$ \\
3 & isopropanol/HCl & $\mathrm{DCM}$ & $92(3: 1)$ \\
4 & $\mathrm{DMPU} / \mathrm{HCl}$ & $\mathrm{DCM}$ & $98(12: 1)$ \\
5 & $\mathrm{DMPU} / \mathrm{HCl}$ & $\mathrm{MeCN}$ & $89(6: 1)$ \\
6 & $\mathrm{DMPU} / \mathrm{HCl}$ & toluene & $88(7: 1)$ \\
7 & $\mathrm{DMPU} / \mathrm{HCl}$ & $\mathrm{THF}$ & $83(5: 1)$ \\
8 & $\mathrm{DMPU} / \mathrm{HCl}$ & $\mathrm{DMF}$ & $78(2: 1)$ \\
9 & $\mathrm{DMPU} / \mathrm{HCl}$ & $\mathrm{DCE}$ & $97(8: 1)$
\end{tabular}

${ }^{a}$ Reaction conditions: 1a $(0.2 \mathrm{mmol}), \mathrm{HCl}$ reagent (0.4 equiv) in solvent $(0.5 \mathrm{~mL}), 0{ }^{\circ} \mathrm{C}$ to rt for $8 \mathrm{~h} .{ }^{b}$ Determined by GC-MS analysis.

gave better yields, higher anti-selectivity and fewer byproducts compared to commercial $\mathrm{HCl}$ reagents (Table 1, entries 1-4). Mixing DMPU/ $\mathrm{HCl}$ with 1a in DCM from $0{ }^{\circ} \mathrm{C}$ to room temperature produced the desired product, chloro-unsaturated ketone 2 , in $98 \%$ yield (Table 1 , entry 4 ). Screening of other solvents indicated that weakly coordinating solvents like DCM, $\mathrm{DCE}$, and toluene were better than more coordinating solvents such as $\mathrm{CH}_{3} \mathrm{CN}$, THF, and DMF (Table 1, entries 5-9).

With the optimized conditions in hand, we explored the substrate scope and functional group tolerance for the hydrochlorination of alkynyl ketones (Table 2, top). We
Table 2. Hydrohalogenation of Ynones ${ }^{a}$
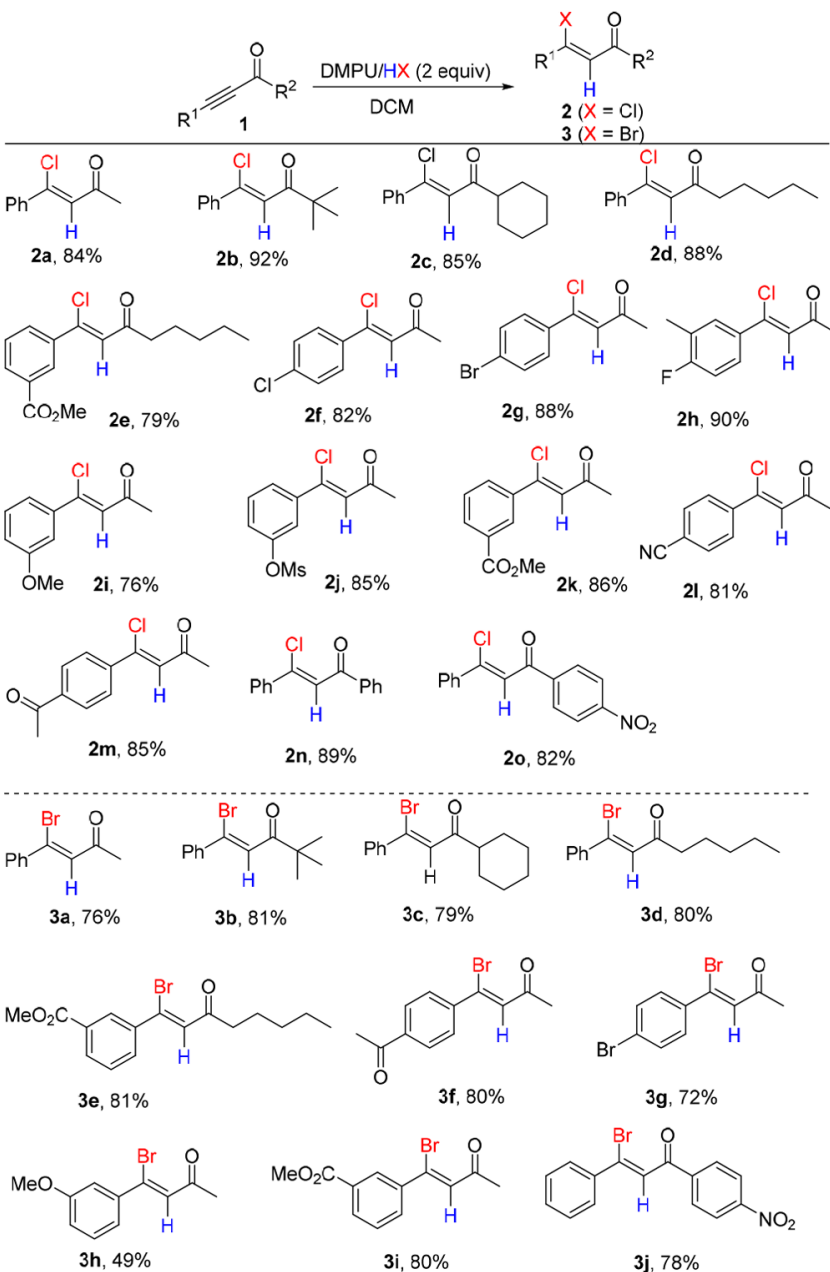

${ }^{a}$ Reaction conditions: ynones $\mathbf{1}(0.2 \mathrm{mmol}), \mathrm{DMPU} / \mathrm{HX}(0.4 \mathrm{mmol})$ in $\mathrm{DCM}(0.5 \mathrm{~mL}), 0^{\circ} \mathrm{C}$ to rt for $8 \mathrm{~h}$; trace syn-addition products were also observed in most case, but they can be easily removed by chromatography.

evaluated the effect of different $\left[R^{1}, R^{2}\right]$ combinations of ynones 1 (Table 2). Combinations of $\left[\mathrm{R}^{1}=\right.$ aryl, $\mathrm{R}^{2}=$ alkyl $]$ (Table 2, $\mathbf{2 a}-\mathbf{1}$ ), $\left[\mathrm{R}^{1}=\right.$ aryl, $\mathrm{R}^{2}=$ aryl $]$ (Table $2, \mathbf{2 n}$ ), $\left[\mathrm{R}^{1}=\right.$ alkyl, $\mathrm{R}^{2}=$ aryl $]$ (Table 2, 2o) worked very well; good chemical yields and high anti-selectivity were observed. The bulky $\mathrm{R}^{2}$ groups (e.g., $t$-Bu, Cy-) did not affect chemical yields (Table 2, 2b, 2c). Equally, the substitution pattern (meta, para) or the electronic properties of the substituents (electron deficient or rich) on $\mathrm{R}^{1}$ or $\mathrm{R}^{2}$ played only a small role; good yields were obtained regardless (Table $2, \mathbf{2 e}-\mathbf{2 m}, \mathbf{2 o}$ ). Various functional groups, such as esters (Table 2, $\mathbf{2 e}, \mathbf{2} \mathbf{k}$ ), ether (Table 2, 2i $\mathbf{i}$ ), halides (Table 2, 2ff, $\mathbf{2 h}$ ), nitrile (Table 2, 2l), nitro (Table 2, $\mathbf{2 o}$ ), and sulfonate (Table 2, 2j), were well tolerated. Similar conditions worked very well for the hydrobromination of ynones when DMPU/HBr was used (Table 2, bottom). In these cases, we observed a very similar reactivity pattern and functional group tolerance (Table $2, \mathbf{3 a}-\mathbf{3} \mathbf{j}$ ). We also tested the hydrohalogenation of dialkyl substituted ynones. Unfortunately we got mostly hydration products and other byproducts. We also attempted to conduct hydroiodination using DMPU-HI reagent, but the preparation of DMPU-HI was not successful (formation of $\mathrm{I}_{2}$ when mixing $\mathrm{HI}$ and DMPU). 
Ynamides are readily available compounds that have found wide applications in organic synthesis. ${ }^{16}$ We were glad to find that our protocol can also be used in the hydrohalogenation of ynamides 4 (Table 3). We first explored the substrate scope

Table 3. Hydrohalogenation of Ynamides ${ }^{a}$

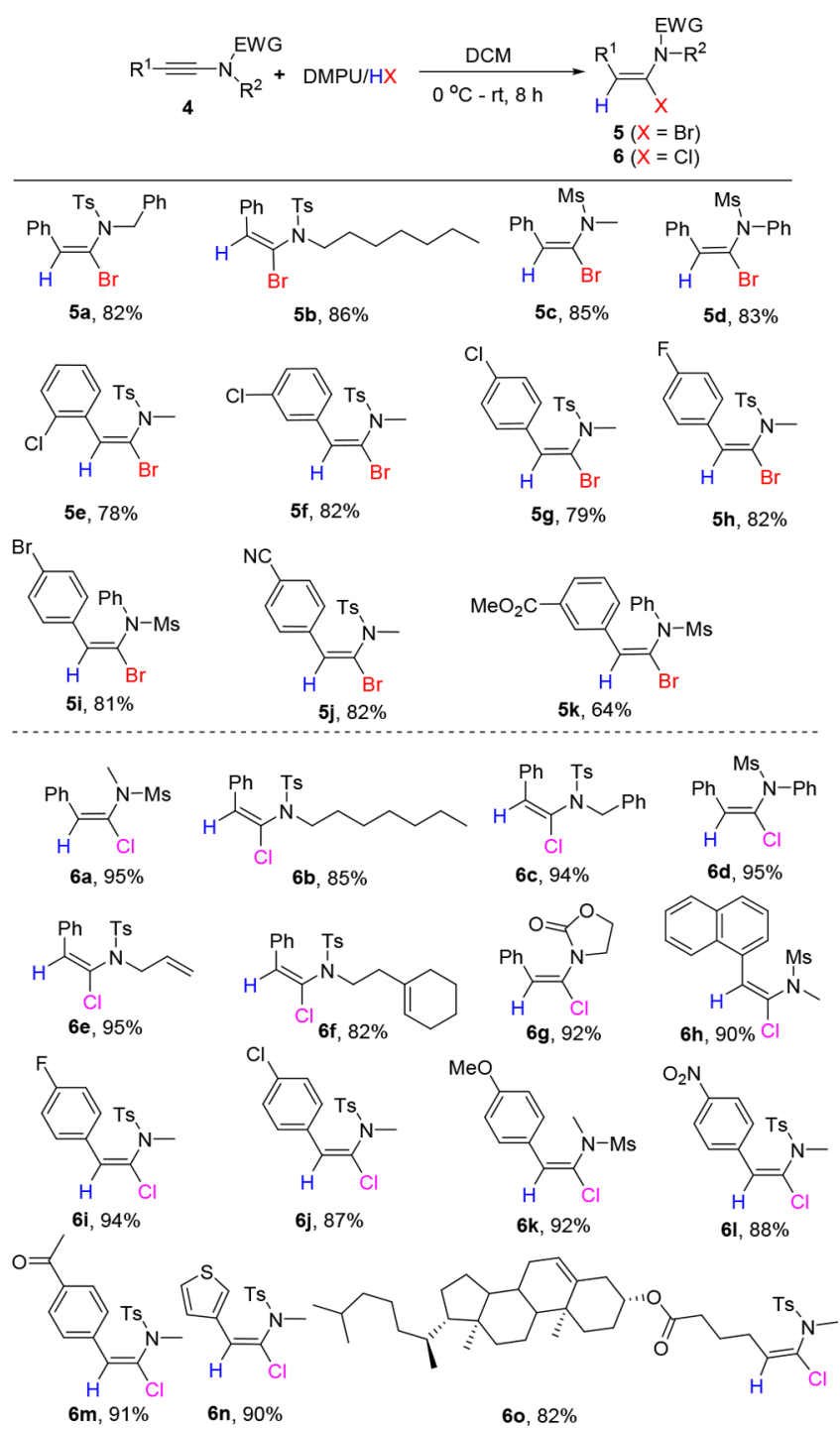

${ }^{a}$ Reaction conditions: ynamide $\mathbf{1}(0.2 \mathrm{mmol}), \mathrm{DMPU} / \mathrm{HX}(0.4 \mathrm{mmol})$ in DCM $(0.5 \mathrm{~mL}), 0{ }^{\circ} \mathrm{C}$ to rt for $8 \mathrm{~h}$.

and functional group tolerance in the hydrobromination of ynamides 4 (Table 3, top). Despite the strong acidity of the DMPU/HBr complex and the fact that ynamides are acid sensitive compounds, the substrate scope was broad: both aliphatic and aromatic substituted ynamides produced synaddition products exclusively in good to excellent yields (Table 3): combinations of $\left[\mathrm{EWG}=\mathrm{Ts}, \mathrm{R}^{2}=\mathrm{Bn}\right]$, $\left[\mathrm{EWG}=\mathrm{Ts}, \mathrm{R}^{2}=n\right.$ heptyl], $\left[\mathrm{EWG}=\mathrm{Ms}, \mathrm{R}^{2}=\right.$ methyl], $\left[\mathrm{EWG}=\mathrm{Ms}, \mathrm{R}^{2}=\right.$ phenyl $]$ all gave good yields (Table $3, \mathbf{5 a}-\mathbf{d}$ ). Also, the substitution pattern (para-, ortho-, or meta-) on the aryl ring of $\mathrm{R}^{1}$ only had a small effect on the efficiency of the reaction, high yields were obtained regardless (Table $3, \mathbf{5 e}-\mathbf{5 i}$ ). It should be noted that acid sensitive functional groups such as nitrile and methyl ester survived our conditions (Table $1, \mathbf{5 j}-\mathbf{5 k}$ ).

Next, we investigated the hydrochlorination of ynamides 4 using $\mathrm{DMPU} / \mathrm{HCl}$ as the chlorination reagent (Table 3, bottom). This protocol also had wide substrate scope and good functional group tolerance (Table 3 bottom). Ynamides 4 with combinations of $\left[\mathrm{EWG}=\mathrm{Ts} / \mathrm{Ms} /\right.$ carbamate, $\mathrm{R}^{2}=$ alkyl, aryl, allyl, homoallyl] delivered 6 in good yields as well as in excellent regio- and stereo-control (Table 3, 6a-g). Interestingly, acid sensitive functional groups, such as alkene, allyl, and carbamate, survived the reaction conditions. We also investigated the effects of the substitution pattern in $\mathrm{R}^{1}$ on the efficiency of reactions. For ynamides with various $\mathrm{R}^{1}$ groups $\left(\mathrm{R}^{1}=\right.$ naphthyl, phenyl rings with electron-donating groups or electron-withdrawing groups, heteroaryls such as thiophene), high yields of $\mathbf{6}$ were obtained regardless (Table 3, 6h-o). Remarkably, ynamides featuring a complex cholesterol scaffold furnished the desired chlorinated product in $82 \%$ yield (Table $3, \mathbf{6 o})$.

Our methodology can be used in larger scale synthesis without complications. The hydrohalogenation of ynone 1a (5 mmol scale) gave chlorinated product $2 \mathbf{a}$ and brominated product $3 \mathrm{a}$ in $85 \%$ and $73 \%$ yields, respectively (Scheme $2 \mathrm{a}$ ). Ynamide 4a (1.0 g scale) was successfully converted to $6 \mathrm{a}$ in $93 \%$ yield (Scheme $2 \mathrm{~b}$ ).

Scheme 2. Gram Scale Hydrohalogenations

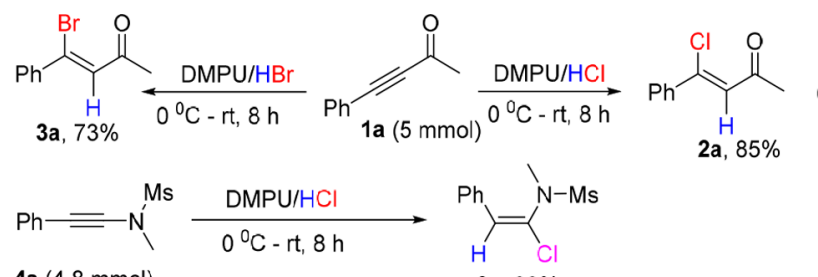

$4 a(4.8 \mathrm{mmol})$ 6a, $93 \%$

Next, we utilized these halogenated alkene products in transition metal catalyzed cross-coupling reactions (Scheme 3).

Scheme 3. Divergent Syntheses from Halogenated Products

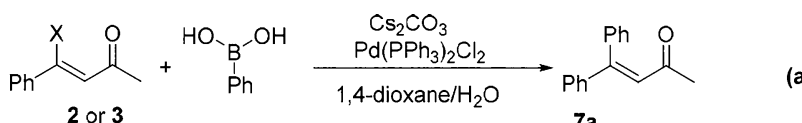

$$
\begin{aligned}
& 2 \text { or } 3 \quad \begin{array}{l}
7 a \\
X=C l, 81 \%
\end{array} \\
& \mathrm{X}=\mathrm{Br}, 90 \% \\
& \underset{6 a}{-\mathrm{N}_{-\mathrm{Ms}}}+\mathrm{PhB}(\mathrm{OH})_{2} \underset{\substack{\mathrm{PtOH} / \mathrm{H}_{2} \mathrm{O}(4 / 1) \\
70^{\circ} \mathrm{C}}}{\mathrm{Pd}(\mathrm{OAc})_{2}, \mathrm{~K}_{2} \mathrm{CO}_{3}} \underset{\mathrm{N}_{\mathrm{Ms}}}{\mathrm{Cl}} \\
& \text { 8a }(90 \%, Z / E=52 / 48) \\
& \underset{5 \mathrm{C}}{-\mathrm{N}_{\mathrm{Ms}}}+\mathrm{TMS}_{\mathrm{Et}_{3} \mathrm{~N} / \mathrm{THF}(1 / 1)}^{\mathrm{Pr}\left(\mathrm{PPh}_{3}\right)_{2} \mathrm{Cl}_{2}, \mathrm{Cul}}
\end{aligned}
$$

Suzuki coupling of halogenated vinyl ketones 2 and 3 with phenyl boronic acid gave good yields of the coupling products (Scheme 3a). Similarly, the Pd catalyzed Suzuki coupling of chlorinated enamide $\mathbf{6 a}$ with phenyl boronic acid (Scheme $3 \mathrm{~b}$ ), and the Sonogashira coupling of brominated enamide $\mathbf{5 c}$ with ethynyltrimethylsilane (Scheme 3c) gave good yields of trisubstituted enamides $\mathbf{8 a}$ and $\mathbf{9 a}$, respectively. On the other hand, copper catalysts, such as $\mathrm{CuCN}$, were not a good catalyst for coupling of $\mathbf{5 c}$.

Our proposed mechanism is shown in Scheme 4. For the hydrohalogenation of ynamides (Scheme 4a), the high acidity 


\section{Scheme 4. Proposed Mechanism ${ }^{a}$}
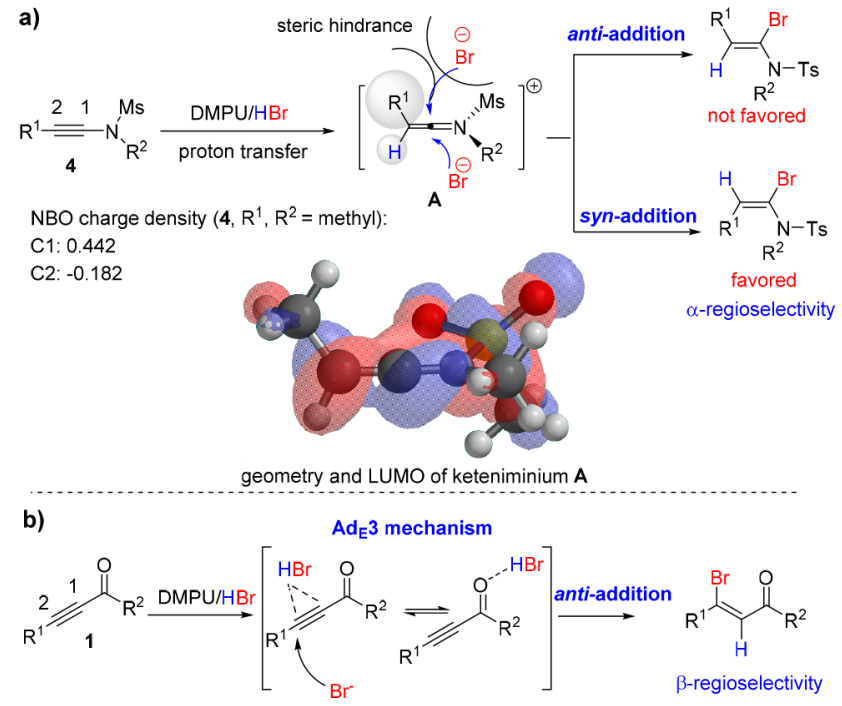

NBO charge density $\left(1, R^{1}, R^{2}=\right.$ methyl):

C1: -0.131

C2: 0.071

O: -0.509

${ }^{a} \mathrm{LUMO}$ of keteniminium was calculated at B3LYP/6-311+G(2df,2p) level of theory.

of DMPU/HX facilitates the rate-determining proton transfer step, which produces the key intermediate, keteniminium $\mathbf{A}^{17}$ Because the nitrogen atom polarizes the triple bond, the regioselectivity of this protonation is mainly determined by the charge distribution on $\mathrm{C} 1$ and $\mathrm{C} 2$ of ynamide 4. Due to the resonance contribution of the lone pair on the nitrogen atom, the NBO charge density of $\mathrm{C} 2$ is significantly higher than $\mathrm{C} 1$, so DMPU/HX should protonate C2 preferentially (Scheme 4a). This pathway eventually leads to the $\alpha$-regioselectivity observed. Also, DFT geometry optimization of $\mathbf{A}$ indicates that the keteniminium intermediate possesses a linear geometry, with its upper face being sterically hindered by the $\mathrm{R}^{1}$ group, thus favoring the nucleophile (bromide/chloride) syn approach (formation of the syn-addition product). ${ }^{11 \mathrm{~b}}$

For the hydrohalogenation of the electron deficient ynone 1, the direct protonation of $\mathbf{1}$ is more difficult, so we proposed a different mechanism (Scheme $4 b$ ). The exclusive formation of the $Z$-isomer (anti-addition) suggested the possibility of an $\operatorname{Ad}_{\mathrm{E}} 3$ mechanism ${ }^{18}$ because the bromide concentration in the reaction medium is not low. ${ }^{19}$ This concerted three-molecule process should furnish the anti-addition product. The regioselectivity found can be rationalized by the fact that the NBO charge density of $\mathrm{C} 1$ is significantly higher than $\mathrm{C} 2$ because of the resonance participation of the carbonyl group (Scheme $4 b$ ). This pathway should eventually lead to the $\beta$-regioselectivity found.

\section{CONCLUSION}

In conclusion, we have developed an atom-economical and metal-free method for the regio- and stereo-selective hydrohalogenation of ynones and ynamides using easily handled $\mathrm{DMPU} / \mathrm{HX}(\mathrm{X}=\mathrm{Br}$ or $\mathrm{Cl})$ reagents. The reaction operates under mild conditions and a range of functional groups are well tolerated. Further applications of these new halogenation reagents are currently underway in our laboratory and will be reported in due course.

\section{EXPERIMENTAL SECTION}

${ }^{1} \mathrm{H}$ NMR (400 or $500 \mathrm{MHz}$ ) and ${ }^{13} \mathrm{C}$ NMR (100 or $125 \mathrm{MHz}$ ) spectra were recorded on a Bruker NMR apparatus. The chemical shifts are reported in $\delta(\mathrm{ppm})$ values $\left({ }^{1} \mathrm{H}\right.$ and ${ }^{13} \mathrm{C}$ NMR relative to $\mathrm{CHCl}_{3}, \delta$ $7.26 \mathrm{ppm}$ for ${ }^{1} \mathrm{H}$ NMR and $\delta 77.0 \mathrm{ppm}$ for ${ }^{13} \mathrm{C}$ NMR). Or alternatively, ${ }^{1} \mathrm{H}$ NMR chemical shifts were referenced to tetramethylsilane signal $(0 \mathrm{ppm})$. Multiplicities are recorded by s (singlet), $\mathrm{d}$ (doublet), $\mathrm{t}$ (triplet), q (quartet), p (pentet), h (hextet), $\mathrm{m}$ (multiplet), and br (broad). Coupling constants $(J)$, are reported in Hertz (Hz). GC analyses were performed using a Shimadzu GC-2010 ultra gas chromatography-mass spectrometry instrument equipped with a Shimadzu AOC-20s autosampler. Commercial reagents and solvents were obtained from the commercial providers and used without further purification. The products were purified using a commercial flash chromatography system or a regular glass column. TLC was developed on silica gel 60 F254 glass plates. The DMPU/HX reagents were prepared using our published procedures. ${ }^{15}$

Methyl 3-(3-oxooct-1-yn-1-yl) Benzoate (1e). ${ }^{20}$ White solid. ${ }^{1} \mathrm{H}$ NMR (400 MHz, chloroform-d) $\delta 8.23(\mathrm{~s}, 1 \mathrm{H}), 8.10(\mathrm{~d}, J=6.6 \mathrm{~Hz}$, $1 \mathrm{H}), 7.73(\mathrm{~d}, J=7.2 \mathrm{~Hz}, 1 \mathrm{H}), 7.47(\mathrm{~s}, 1 \mathrm{H}), 3.93(\mathrm{~s}, 3 \mathrm{H}), 2.66(\mathrm{t}, J=$ $7.2 \mathrm{~Hz}, 2 \mathrm{H}), 1.91-1.67(\mathrm{~m}, 2 \mathrm{H}), 1.35(\mathrm{~d}, J=3.3 \mathrm{~Hz}, 4 \mathrm{H}), 0.91(\mathrm{t}, J=$ $7.5 \mathrm{~Hz}, 3 \mathrm{H}) .{ }^{13} \mathrm{C}\left\{{ }^{1} \mathrm{H}\right\}$ NMR (100 MHz, chloroform-d) $\delta 187.9,165.8$, 136.9, 133.9, 131.3, 130.8, 128.7, 120.6, 88.8, 88.2, 52.5, 45.5, 31.1, 23.8, 22.3, 13.8. HRMS (ESI-FT-ICR) calcd. for $\mathrm{C}_{16} \mathrm{H}_{19} \mathrm{O}_{3}[\mathrm{M}+\mathrm{H}]^{+}$: 259.1334, found: 259.1327 .

4-(4-Fluoro-3-methylphenyl)but-3-yn-2-one (1h). Yellow solid. Mp $42-43{ }^{\circ} \mathrm{C} .{ }^{1} \mathrm{H}$ NMR (400 MHz, chloroform- $d$ ) $\delta 7.61-$ $7.33(\mathrm{~m}, 2 \mathrm{H}), 7.00(\mathrm{t}, J=8.8 \mathrm{~Hz}, 1 \mathrm{H}), 2.42(\mathrm{~s}, 3 \mathrm{H}), 2.26(\mathrm{~s}, 3 \mathrm{H}) .{ }^{19} \mathrm{~F}$ NMR (376 MHz, chloroform-d) $\delta-109.52--112.87(\mathrm{~m}) .{ }^{13} \mathrm{C}\left\{{ }^{1} \mathrm{H}\right\}$ NMR (100 MHz, chloroform- $d$ ) $\delta 184.4,162.7(\mathrm{~d}, J=252.4 \mathrm{~Hz})$, $136.5,132.7$ (d, $J=8.9 \mathrm{~Hz}), 125.9$ (d, $J=18.2 \mathrm{~Hz}), 115.8,115.6,89.7$, 87.9, $32.6(\mathrm{~d}, J=5.3 \mathrm{~Hz}), 14.3$. HRMS (ESI-FT-ICR) calcd. for $\mathrm{C}_{12} \mathrm{H}_{10} \mathrm{FO}[\mathrm{M}+\mathrm{H}]^{+}: 177.0710$, found: 177.0703 .

3-(3-Oxobut-1-yn-1-yl)phenyl methanesulfonate $(1 \mathrm{j}) .^{20}$ White solid. ${ }^{1} \mathrm{H}$ NMR (400 MHz, chloroform- $d$ ) $\delta$ 7.57-7.34 (m, $4 \mathrm{H}), 3.17$ (s, 3H), $2.44(\mathrm{~s}, 3 \mathrm{H}) .{ }^{13} \mathrm{C}\left\{{ }^{1} \mathrm{H}\right\}$ NMR $(100 \mathrm{MHz}$, chloroform-d) $\delta 184.1,148.8,131.8,130.4,126.2,124.6,122.0,88.9$, 87.6, 37.7, 32.7. HRMS(ESI-FT-ICR) calcd. for $\mathrm{C}_{11} \mathrm{H}_{11} \mathrm{SO}_{4}[\mathrm{M}+\mathrm{H}]^{+}$: 239.0378, found:239.0371.

Methyl 3-(3-oxobut-1-yn-1-yl)benzoate (1k). ${ }^{20}$ White solid. ${ }^{1} \mathrm{H}$ NMR (400 MHz, chloroform-d) $\delta 8.22(\mathrm{~s}, 1 \mathrm{H}), 8.12-8.00(\mathrm{~m}$, $1 \mathrm{H}), 7.79-7.64(\mathrm{~m}, 1 \mathrm{H}), 7.46-7.42(\mathrm{~m}, 1 \mathrm{H}), 3.92(\mathrm{~s}, 3 \mathrm{H}), 2.44(\mathrm{~s}$, $3 \mathrm{H}) .{ }^{13} \mathrm{C}\left\{{ }^{1} \mathrm{H}\right\}$ NMR (100 MHz, chloroform-d) $\delta 184.2,165.8,136.8$, 134.0, 131.5, 130.8, 128.8, 120.4, 88.6, 88.5, 52.4, 32.7. HRMS (ESIFT-ICR) calcd. for $\mathrm{C}_{12} \mathrm{H}_{11} \mathrm{O}_{3}[\mathrm{M}+\mathrm{H}]^{+}$: 203.0708, found:203.0720.

4-(3-Oxobut-1-yn-1-yl)benzonitrile (1I). ${ }^{20}$ White solid. ${ }^{1} \mathrm{H}$ NMR (400 MHz, chloroform- $d$ ) $\delta 7.66-7.63(\mathrm{~m}, 4 \mathrm{H}), 2.46(\mathrm{~s}$, $3 \mathrm{H}) .{ }^{13} \mathrm{C}\left\{{ }^{1} \mathrm{H}\right\}$ NMR $(100 \mathrm{MHz}$, chloroform-d) $\delta 183.9,133.2,132.2$, 124.7, 117.8, 113.9, 90.5, 86.8, 32.7. HRMS (ESI-FT-ICR) calcd. for $\mathrm{C}_{11} \mathrm{H}_{8} \mathrm{NO}[\mathrm{M}+\mathrm{H}]^{+}: 170.0606$, found:170.0600.

4-(4-Acetylphenyl)but-3-yn-2-one $(1 \mathrm{~m}){ }^{20}$ White solid. ${ }^{1} \mathrm{H}$ NMR $(400 \mathrm{MHz}$, chloroform-d) $\delta 7.93(\mathrm{~d}, J=8.2 \mathrm{~Hz}, 2 \mathrm{H}), 7.62$ $(\mathrm{d}, J=8.2 \mathrm{~Hz}, 2 \mathrm{H}), 2.59(\mathrm{~s}, 3 \mathrm{H}), 2.44(\mathrm{~s}, 3 \mathrm{H}) \cdot{ }^{13} \mathrm{C}\left\{{ }^{1} \mathrm{H}\right\} \mathrm{NMR}(100$ MHz, chloroform-d) $\delta 196.9,184.2,138.0,133.0,128.3,124.5,89.9$, 88.3, 32.7, 32., 26.64. HRMS (ESI-FT-ICR) calcd. for $\mathrm{C}_{12} \mathrm{H}_{11} \mathrm{O}_{2}[\mathrm{M}$ $+\mathrm{H}]^{+}: 187.0759$, found:187.0753.

Synthesis of Ynamides 4. General Procedure. To a mixture of alkynyl bromide $(3.0 \mathrm{mmol}), 1,10$-phenanthroline $(0.6 \mathrm{mmol}, 108$ $\mathrm{mg}), \mathrm{CuSO}_{4} \cdot 5 \mathrm{H}_{2} \mathrm{O}(0.3 \mathrm{mmol}, 149 \mathrm{mg})$, and $\mathrm{K}_{2} \mathrm{CO}_{3}(6.0 \mathrm{mmol}, 828$ $\mathrm{mg})$ in dry toluene $(15.0 \mathrm{~mL})$, amide $(3.6 \mathrm{~mol})$ was added. The reaction mixture was stirred at $70{ }^{\circ} \mathrm{C}$ under the nitrogen atmosphere. The progress of the reaction was monitored periodically by TLC. After the completion of the reaction, the reaction mixture was filtered through a pad of Celite and the solvent was removed in vacuum. The resulting residue was purified by chromatography on silica gel to give the desired products.

$\mathrm{N}$-(2-(Cyclohex-1-en-1-yl)ethyl)-4-methyl- $\mathrm{N}$-(phenylethynyl)benzenesulfonamide (4f). Pale yellow oil. ${ }^{1} \mathrm{H}$ NMR $(500 \mathrm{MHz}$, chloroform-d) $\delta 7.86(\mathrm{~d}, J=8.3 \mathrm{~Hz}, 2 \mathrm{H}), 7.46-7.34(\mathrm{~m}, 4 \mathrm{H}), 7.35-$ 
$7.26(\mathrm{~m}, 3 \mathrm{H}), 5.48(\mathrm{~s}, 1 \mathrm{H}), 3.65-3.34(\mathrm{~m}, 2 \mathrm{H}), 2.47(\mathrm{~s}, 3 \mathrm{H}), 2.33(\mathrm{t}, J$ $=7.6 \mathrm{~Hz}, 2 \mathrm{H}), 1.99-1.94(\mathrm{~m}, 4 \mathrm{H}), 1.73-1.59(\mathrm{~m}, 2 \mathrm{H}), 1.57-1.52(\mathrm{~m}$, $2 \mathrm{H}) .{ }^{13} \mathrm{C}\left\{{ }^{1} \mathrm{H}\right\}$ NMR $(100 \mathrm{MHz}$, chloroform- $d) \delta 144.5,134.8,133.3$, $131.3,129.7,128.3,127.7,124.2,123.0,82.5,70.8,50.3,36.4,28.2$, 25.3, 22.8, 22.2, 21.7. HRMS (ESI-FT-ICR) calcd. for $\mathrm{C}_{23} \mathrm{H}_{26} \mathrm{NO}_{2} \mathrm{~S}$ $[\mathrm{M}+\mathrm{H}]^{+}: 380.1684$, found: 380.1678 .

(3S, 10R, 13R, 17R)-10,13-Dimethyl-17-((R)-6-methylheptan-2-yl)$2,3,4,7,8,9,10,11,12,13,14,15,16,17$-tetradecahydro-1H-cyclopenta[a]phenanthren-3-yl 6-((N,4-dimethylphenyl)sulfonamido)hex-5ynoate (4o). White solid. Mp 94-95 ${ }^{\circ} \mathrm{C} .{ }^{1} \mathrm{H}$ NMR $(400 \mathrm{MHz}$, chloroform- $d) \delta 7.76(\mathrm{~d}, J=8.3 \mathrm{~Hz}, 2 \mathrm{H}), 7.34(\mathrm{~d}, J=7.9 \mathrm{~Hz}, 2 \mathrm{H})$, $5.35(\mathrm{~d}, J=4.9 \mathrm{~Hz}, 1 \mathrm{H}), 4.62-4.60(\mathrm{~m}, 1 \mathrm{H}), 2.99(\mathrm{~s}, 3 \mathrm{H}), 2.43(\mathrm{~s}$, $3 \mathrm{H}), 2.35-2.31(\mathrm{~m}, 6 \mathrm{H}), 2.07-1.05(\mathrm{~m}, 28 \mathrm{H}), 1.00(\mathrm{~s}, 3 \mathrm{H}), 0.89(\mathrm{~d}, J$ $=6.5 \mathrm{~Hz}, 3 \mathrm{H}), 0.85(\mathrm{~d}, J=1.8 \mathrm{~Hz}, 3 \mathrm{H}), 0.83(\mathrm{~d}, J=1.8 \mathrm{~Hz}, 3 \mathrm{H}), 0.66$ $(\mathrm{s}, 3 \mathrm{H}) .{ }^{13} \mathrm{C}\left\{{ }^{1} \mathrm{H}\right\}$ NMR $(100 \mathrm{MHz},) \delta 172.5,144.5,139.6,133.1,129.7$, 127.8 , 127.7, 122.6, 75.6, 74.0, 67.4, 56.7, 56.1, 50.0, 42.3, 39.7, 39.5, $38.1,37.0,36.6,36.2,35.8,33.4,31.9,31.8,28.2,28.0,27.8,24.2,23.8$, 22.8, 22.5, 21.7, 21.0, 19.3, 18.7, 17.9, 11.8. HRMS (ESI ${ }^{+}$) calcd. for $\mathrm{C}_{41} \mathrm{H}_{61} \mathrm{NO}_{4} \mathrm{~S}[\mathrm{M}+\mathrm{H}]^{+}:$664.4400, found: 664.4385 .

Hydrochlorination of Ynones 1. To a solution of ynone $1(0.2$ $\mathrm{mmol})$ in DCM $(0.5 \mathrm{~mL})$ was added DMPU/HCl $(43 \mathrm{wt} / \mathrm{wt} \%)(34$ $\mathrm{mg}, 0.4 \mathrm{mmol}$ ) at $0{ }^{\circ} \mathrm{C}$, then the mixture was warmed to room temperature and was stirred for $8 \mathrm{~h}$. After completion, the solvent was evaporated under the reduced pressure and the residue was purified by silica gel column chromatography (hexanes/EtOAc as elute solvent) to afford the corresponding products 2 .

(Z)-4-Chloro-4-phenylbut-3-en-2-one $(2 a) .^{3 e}$ Purified by flash column chromatography (ethyl acetate/hexanes $=1: 20$ ) give the product as colorless oil, $30.2 \mathrm{mg} 84 \%$ yield. ${ }^{1} \mathrm{H}$ NMR $(400 \mathrm{MHz}$, chloroform- $d) \delta 7.86-7.55(\mathrm{~m}, 2 \mathrm{H}), 7.58-7.31(\mathrm{~m}, 4 \mathrm{H}), 6.77(\mathrm{~s}, 1 \mathrm{H})$, $2.47(\mathrm{~s}, 3 \mathrm{H}) .{ }^{13} \mathrm{C}\left\{{ }^{1} \mathrm{H}\right\}$ NMR $(100 \mathrm{MHz}$, chloroform- $d) \delta 196.7,143.1$, 137.3, 130.7, 128.6, 127.3, 124.7, 31.9.

(Z)-1-Chloro-4,4-dimethyl-1-phenylpent-1-en-3-one (2b). ${ }^{21}$ Purified by flash column chromatography (ethyl acetate/hexanes = 1:20)give the product as yellow oil, $40.8 \mathrm{mg}, 92 \%$ yield. ${ }^{1} \mathrm{H}$ NMR $(400 \mathrm{MHz}$, chloroform- $d) \delta 7.77-7.56(\mathrm{~m}, 2 \mathrm{H}), 7.59-7.25(\mathrm{~m}, 3 \mathrm{H})$, $7.06(\mathrm{~s}, 1 \mathrm{H}), 1.22(\mathrm{~s}, 9 \mathrm{H}) .{ }^{13} \mathrm{C}\left\{{ }^{1} \mathrm{H}\right\}$ NMR $(100 \mathrm{MHz}$, chloroform- $d) \delta$ 203.6, 143.2, 137.8, 130.4, 128.6, 127.2, 119.9, 44.5, 26.3. HRMS (ESIFT-ICR) calcd. for $\mathrm{C}_{13} \mathrm{H}_{16} \mathrm{ClO}[\mathrm{M}+\mathrm{H}]^{+}$: 223.0890, found: 223.0885 .

(Z)-3-Chloro-1-cyclohexyl-3-phenylprop-2-en-1-one (2c). ${ }^{22}$ Purified by flash column chromatography (ethyl acetate/hexanes = 1:20)give the product as yellow oil, $42.1 \mathrm{mg}, 85 \%$ yield. ${ }^{1} \mathrm{H}$ NMR $(400 \mathrm{MHz}$, chloroform- $d) \delta 7.90-7.51(\mathrm{~m}, 2 \mathrm{H}), 7.41-7.36(\mathrm{~m}, 3 \mathrm{H})$, $6.84(\mathrm{~s}, 1 \mathrm{H}), 2.58-2.54(\mathrm{~m}, 1 \mathrm{H}), 1.92-1.90(\mathrm{~m}, 2 \mathrm{H}), 1.80-1.78(\mathrm{~m}$, $2 \mathrm{H}), 1.67-1.65(\mathrm{~m}, 1 \mathrm{H}), 1.50-1.05(\mathrm{~m}, 5 \mathrm{H}) .{ }^{13} \mathrm{C}\left\{{ }^{1} \mathrm{H}\right\}$ NMR $(100$ $\mathrm{MHz}$, chloroform- $d$ ) $\delta 201.5,142.6,137.55,130.47,128.57,127.24$, 122.66, 51.63, 28.35, 25.85, 25.65. HRMS (ESI-FT-ICR) calcd. for $\mathrm{C}_{15} \mathrm{H}_{18} \mathrm{ClO}[\mathrm{M}+\mathrm{H}]^{+}: 249.1046$, found: 249.1039 .

(Z)-1-Chloro-1-phenyloct-1-en-3-one (2d). Purified by flash column chromatography (ethyl acetate/hexanes $=1: 20$ ), yellow oil, $41.5 \mathrm{mg}, 88 \%$ yield. ${ }^{1} \mathrm{H}$ NMR ( $400 \mathrm{MHz}$, chloroform- $d$ ) $\delta 7.67-7.75$ $(\mathrm{m}, 2 \mathrm{H}), 7.50-7.30(\mathrm{~m}, 3 \mathrm{H}), 6.78(\mathrm{~s}, 1 \mathrm{H}), 2.68(\mathrm{t}, J=7.4 \mathrm{~Hz}, 2 \mathrm{H})$, $1.67-1.63(\mathrm{~m}, 2 \mathrm{H}), 1.33-1.30(\mathrm{~m}, 4 \mathrm{H}), 0.90(\mathrm{t}, J=6.8 \mathrm{~Hz}, 3 \mathrm{H})$. ${ }^{13} \mathrm{C}\left\{{ }^{1} \mathrm{H}\right\}$ NMR (100 MHz, chloroform- $d$ ) $\delta 199.01,142.31,137.44$, $130.53,128.59,127.26,123.86,44.44,31.38,23.68,22.48,13.92$. HRMS (ESI-FT-ICR) calcd. for $\mathrm{C}_{14} \mathrm{H}_{18} \mathrm{ClO}[\mathrm{M}+\mathrm{H}]^{+}:$237.1046, found: 237.1040 .

Methyl (Z)-3-(1-Chloro-3-oxooct-1-en-1-yl)benzoate (2e). Purified by flash column chromatography (ethyl acetate/hexanes $=1 / 20$ ), yellow oil. NMR (400 MHz, chloroform- $d$ ) $\delta 8.31(\mathrm{~s}, 1 \mathrm{H}), 8.07(\mathrm{~d}, J=$ $7.1 \mathrm{~Hz}, 1 \mathrm{H}), 7.85(\mathrm{~d}, J=7.1 \mathrm{~Hz}, 1 \mathrm{H}), 7.48(\mathrm{t}, J=7.8 \mathrm{~Hz}, 1 \mathrm{H}), 6.83(\mathrm{~s}$, $1 \mathrm{H}), 3.93(\mathrm{~s}, 3 \mathrm{H}), 2.68(\mathrm{t}, J=7.3 \mathrm{~Hz}, 2 \mathrm{H}), 1.87-1.59(\mathrm{~m}, 2 \mathrm{H}), 1.35-$ $1.29(\mathrm{~m}, 4 \mathrm{H}), 0.88(\mathrm{t}, J=6.6 \mathrm{~Hz}, 3 \mathrm{H}) .{ }^{13} \mathrm{C}\left\{{ }^{1} \mathrm{H}\right\}$ NMR $(100 \mathrm{MHz}$, chloroform- $d$ ) $\delta 198.8,166.2,140.9,137.8,131.5,131.4,130.7,128.8$, 128.2, 124.8, 52.4, 44.5, 31.4, 23.6, 22.5, 13.9. HRMS (ESI-FT-ICR) calcd. for $\mathrm{C}_{16} \mathrm{H}_{20} \mathrm{ClO}_{3}[\mathrm{M}+\mathrm{H}]^{+}$: 295.1101, found: 295.1094.

(Z)-4-Chloro-4-(4-chlorophenyl)but-3-en-2-one (2f). Purified by flash column chromatography (ethyl acetate/hexane $=1: 20$ ) give the product as pale yellow oil, $35 \mathrm{mg}, 82 \%$ yield. ${ }^{1} \mathrm{H}$ NMR $(400 \mathrm{MHz}$, chloroform- $d$ ) $\delta 7.60(\mathrm{~d}, J=8.6 \mathrm{~Hz}, 2 \mathrm{H}), 7.37(\mathrm{~d}, J=8.6 \mathrm{~Hz}, 2 \mathrm{H})$, $6.73(\mathrm{~s}, 1 \mathrm{H}), 2.46(\mathrm{~s}, 3 \mathrm{H}) \cdot{ }^{13} \mathrm{C}\left\{{ }^{1} \mathrm{H}\right\}$ NMR (100 MHz, chloroform- $\left.d\right) \delta$ $196.3,141.6,136.9,135.7,128.9,128.5,124.9,31.9$. HRMS (ESI-FTICR) calcd. for $\mathrm{C}_{10} \mathrm{H}_{9} \mathrm{Cl}_{2} \mathrm{O}[\mathrm{M}+\mathrm{H}]^{+}$: 215.0030, found: 215.0024 .

(Z)-4-(4-Bromophenyl)-4-chlorobut- 3-en-2-one (2g). Purified by flash column chromatography (ethyl acetate/hexanes $=1: 20$ ), yellow solid, $44.8 \mathrm{mg}, 88 \%$ yield. Mp $42-43{ }^{\circ} \mathrm{C}{ }^{1} \mathrm{H}$ NMR $(400 \mathrm{MHz}$, chloroform- $d$ ) $\delta 7.53(\mathrm{~s}, 4 \mathrm{H}), 6.74(\mathrm{~s}, 1 \mathrm{H}), 2.45(\mathrm{~s}, 3 \mathrm{H}) .{ }^{13} \mathrm{C}\left\{{ }^{1} \mathrm{H}\right\}$ NMR (100 MHz, chloroform-d) $\delta 196.7,141.7,136.1,131.9,128.7$, 125.2, 124.9, 31.9. HRMS (ESI-FT-ICR) calcd. for $\mathrm{C}_{10} \mathrm{H}_{7} \mathrm{ClBrO}[\mathrm{M}-$ $\mathrm{H}^{+}$: 256.9369 , found: 256.9362 .

(Z)-4-Chloro-4-(4-fluoro-3-methylphenyl)but-3-en-2-one (2h). Purified by flash column chromatography (ethyl acetate/hexanes = 1:20), yellow oil, $38.1 \mathrm{mg}$, 90\% yield. ${ }^{1} \mathrm{H}$ NMR (400 MHz, chloroform- $d) \delta 7.48-7.42(\mathrm{~m}, 2 \mathrm{H}), 7.01(\mathrm{t}, J=8.8 \mathrm{~Hz}, 1 \mathrm{H}), 6.69$ $(\mathrm{s}, 1 \mathrm{H}), 2.44(\mathrm{~s}, 3 \mathrm{H}), 2.28(\mathrm{~s}, 3 \mathrm{H}) .{ }^{19} \mathrm{~F}$ NMR $(376 \mathrm{MHz}$, chloroformd) $\delta-101.3--135.0(\mathrm{~m}, 1 \mathrm{~F}) .{ }^{13} \mathrm{C}\left\{{ }^{1} \mathrm{H}\right\}$ NMR $(100 \mathrm{MHz}$, chloroform-d) $\delta$ 196.4, $162.7(\mathrm{~d}, J=250.8 \mathrm{~Hz}), 142.1,133.1$ (d, $J=$ $3.8 \mathrm{~Hz}), 130.7(\mathrm{~d}, J=6.1 \mathrm{~Hz}), 126.7(\mathrm{~d}, J=8.7 \mathrm{~Hz}), 125$. Four $(\mathrm{d}, J=$ $17.9 \mathrm{~Hz}), 124.3,115.3(\mathrm{~d}, J=23.1 \mathrm{~Hz}), 31.9,14.6(\mathrm{~d}, J=3.3 \mathrm{~Hz})$. HRMS (ESI-FT-ICR) calcd. for $\mathrm{C}_{11} \mathrm{H}_{11} \mathrm{ClFO}[\mathrm{M}+\mathrm{H}]^{+}:$213.0482, found:213.0477.

(Z)-4-Chloro-4-(3-methoxyphenyl)but-3-en-2-one (2i). Purified by flash column chromatography (ethyl acetate/hexanes $=1: 20$ ), yellow oil, $31.9 \mathrm{mg}, 76 \%$ yield. ${ }^{1} \mathrm{H}$ NMR (400 MHz, chloroform- $d$ ) $7.29(\mathrm{t}, J=$ $7.9 \mathrm{~Hz}, 1 \mathrm{H}), 7.23(\mathrm{~d}, J=7.8 \mathrm{~Hz}, 1 \mathrm{H}), 7.18(\mathrm{~s}, 1 \mathrm{H}), 7.00-6.91(\mathrm{~m}$, $1 \mathrm{H}), 6.75(\mathrm{~s}, 1 \mathrm{H}), 3.82(\mathrm{~s}, 3 \mathrm{H}), 2.45(\mathrm{~s}, 3 \mathrm{H}) .{ }^{13} \mathrm{C}\left\{{ }^{1} \mathrm{H}\right\}$ NMR $(100$ $\mathrm{MHz}$, chloroform- $d$ ) $\delta$ 196.6, 159.6, 142.7, 138.7, 129.6, 124.9, 119.6, 116.3, 112.9, 55.4, 31.9. HRMS (ESI-FT-ICR) calcd. for $\mathrm{C}_{11} \mathrm{H}_{12} \mathrm{ClO}_{2}$ $[\mathrm{M}+\mathrm{H}]^{+}:$211.0526, found: 211.0519 .

(Z)-3-(1-Chloro-3-oxobut-1-en-1-yl)phenyl methanesulfonate (2j). Purified by flash column chromatography (ethyl acetate/hexanes $=1: 10$ ), pale red oil, $46.5 \mathrm{mg}, 85 \%$ yield. ${ }^{1} \mathrm{H}$ NMR $(400 \mathrm{MHz}$, chloroform- $d) \delta 7.63(\mathrm{~d}, J=7.9 \mathrm{~Hz}, 1 \mathrm{H}), 7.58(\mathrm{~s}, 1 \mathrm{H}), 7.46(\mathrm{t}, J=8.0$ $\mathrm{Hz}, 1 \mathrm{H}), 7.36(\mathrm{ddd}, J=8.1,2.5,1.1 \mathrm{~Hz}, 1 \mathrm{H}), 6.77(\mathrm{~s}, 1 \mathrm{H}), 3.18(\mathrm{~s}$, $3 \mathrm{H}), 2.46(\mathrm{~s}, 3 \mathrm{H}) .{ }^{13} \mathrm{C}\left\{{ }^{1} \mathrm{H}\right\}$ NMR $(100 \mathrm{MHz}$, chloroform- $d) \delta 196.3$, $149.1,140.6,139.4,130.3,126.1,125.8,124.1,121.1,37.7,31.9$. HRMS (ESI-FT-ICR) calcd. for $\mathrm{C}_{11} \mathrm{H}_{12} \mathrm{ClSO}_{4}[\mathrm{M}+\mathrm{H}]^{+}:$275.0145, found:275.0140.

Methyl (Z)-3-(1-Chloro-3-oxobut-1-en-1-yl)benzoate (2k). Purified by flash column chromatography (ethyl acetate/hexanes $=1: 15$ ), yellow solid, $40.9 \mathrm{mg}$, $86 \%$ yield. Mp $44-45{ }^{\circ} \mathrm{C}{ }^{1} \mathrm{H}$ NMR $(400 \mathrm{MHz}$, chloroform- $d) \delta 8.31(\mathrm{~s}, 1 \mathrm{H}), 8.08(\mathrm{~d}, J=7.7 \mathrm{~Hz}, 1 \mathrm{H}), 7.85(\mathrm{~d}, J=8.9$ $\mathrm{Hz}, 1 \mathrm{H}), 7.49(\mathrm{t}, J=7.8 \mathrm{~Hz}, 1 \mathrm{H}), 6.82(\mathrm{~s}, 1 \mathrm{H}), 3.93(\mathrm{~s}, 3 \mathrm{H}), 2.47(\mathrm{~s}$ $3 \mathrm{H}) .{ }^{13} \mathrm{C}\left\{{ }^{1} \mathrm{H}\right\}$ NMR $(100 \mathrm{MHz}$, chloroform-d) $\delta 196.4,166.1,141.6$, $137.6,131.5,130.7,128.9,128.2,125.4,52.4$, 31.9. HRMS (ESI-FTICR) calcd. for $\mathrm{C}_{12} \mathrm{H}_{12} \mathrm{ClO}_{3}[\mathrm{M}+\mathrm{H}]^{+}: 239.0475$, found:239.0470.

(Z)-4-(1-Chloro-3-oxobut-1-en-1-yl)benzonitrile (2I). Purified by flash column chromatography (ethyl acetate/hexanes $=1: 10$ ), yellow solid, $33.2 \mathrm{mg}, 81 \%$ yield. $\mathrm{Mp} 76-78{ }^{\circ} \mathrm{C} .{ }^{1} \mathrm{H}$ NMR $(400 \mathrm{MHz}$, chloroform-d) $\delta 7.77(\mathrm{~d}, J=8.3 \mathrm{~Hz}, 2 \mathrm{H}), 7.70(\mathrm{~d}, J=8.3 \mathrm{~Hz}, 2 \mathrm{H})$, $6.79(\mathrm{~s}, 1 \mathrm{H}), 2.47(\mathrm{~s}, 3 \mathrm{H}) .{ }^{13} \mathrm{C}\left\{{ }^{1} \mathrm{H}\right\} \mathrm{NMR}(100 \mathrm{MHz}$, chloroform- $d) \delta$ 196.1, 141.4, 140.2, 132.4, 127.9, 126.8, 117.9, 114.1, 31.8. HRMS (ESI-FT-ICR) calcd. for $\mathrm{C}_{11} \mathrm{H}_{9} \mathrm{ClNO}[\mathrm{M}+\mathrm{H}]^{+}:$206.0373, found: 206.0366.

(Z)-4-(4-Acetylphenyl)-4-chlorobut-3-en-2-one ( $2 m)$. Purified by flash column chromatography (ethyl acetate/hexanes $=1: 15$ ), yellow oil, $37.4 \mathrm{mg}, 85 \%$ yield. ${ }^{1} \mathrm{H}$ NMR (400 MHz, chloroform- $d$ ) 7.97 (d, J $=8.5 \mathrm{~Hz}, 2 \mathrm{H}), 7.75(\mathrm{~d}, J=8.6 \mathrm{~Hz}, 2 \mathrm{H}), 6.81(\mathrm{~s}, 1 \mathrm{H}), 2.61(\mathrm{~s}, 3 \mathrm{H})$, $2.48(\mathrm{~s}, 3 \mathrm{H}) .{ }^{13} \mathrm{C}\left\{{ }^{1} \mathrm{H}\right\}$ NMR $(100 \mathrm{MHz}$, chloroform- $d) \delta 197.1,196.4$, $141.3,138.4,128.5,127.5,126.2,31.9,26.7$. HRMS (ESI-FT-ICR) calcd. for $\mathrm{C}_{12} \mathrm{H}_{12} \mathrm{ClO}_{2}[\mathrm{M}+\mathrm{H}]^{+}$: 223.0526, found: 223.0520 .

(Z)-3-Chloro-1,3-diphenylprop-2-en-1-one (2n). Purified by flash column chromatography (ethyl acetate $/$ hexanes $=1: 20$ ), yellow oil, 43 $\mathrm{mg}, 89 \%$ yield. ${ }^{1} \mathrm{H}$ NMR ( $500 \mathrm{MHz}$, chloroform- $d$ ) $\delta 8.02$ (d, $J=7.0$ $\mathrm{Hz}, 2 \mathrm{H}), 7.83-7.72(\mathrm{~m}, 2 \mathrm{H}), 7.60(\mathrm{t}, J=7.3 \mathrm{~Hz}, 1 \mathrm{H}), 7.55-7.42(\mathrm{~m}$, $5 \mathrm{H}), 7.37(\mathrm{~s}, 1 \mathrm{H}) .{ }^{13} \mathrm{C}\left\{{ }^{1} \mathrm{H}\right\}$ NMR $(126 \mathrm{MHz}$, chloroform- $d) \delta 189.8$, 143.3, 133.4, 130.6, 128.7, 128.7, 128.7, 127.2, 121.5. HRMS (ESI-FTICR) calcd. for $\mathrm{C}_{15} \mathrm{H}_{12} \mathrm{ClO}[\mathrm{M}+\mathrm{H}]^{+}$: 243.0577, found: 243.0569 .

(Z)-3-Chloro-1-(4-nitrophenyl)-3-phenylprop-2-en-1-one (2o). ${ }^{3 e}$ Purified by flash column chromatography (ethyl acetate/hexanes = 
1:15), yellow oil, $47 \mathrm{mg}, 82 \%$ yield. ${ }^{1} \mathrm{H}$ NMR ( $500 \mathrm{MHz}$, chloroformd) ${ }^{1} \mathrm{H}$ NMR (500 MHz, chloroform-d) $\delta 8.34$ (d, $\left.J=8.9 \mathrm{~Hz}, 2 \mathrm{H}\right), 8.14$ $(\mathrm{d}, J=8.9 \mathrm{~Hz}, 2 \mathrm{H}), 7.79(\mathrm{dt}, J=6.7,1.6 \mathrm{~Hz}, 2 \mathrm{H}), 7.61-7.41(\mathrm{~m}, 3 \mathrm{H})$, $7.37(\mathrm{~s}, 1 \mathrm{H})$. HRMS (ESI-FT-ICR) calcd. for $\mathrm{C}_{15} \mathrm{H}_{11} \mathrm{ClNO}_{3}[\mathrm{M}+\mathrm{H}]^{+}$: 288.0427, found: 288.0419 .

Hydrobromination of Ynones 1. To a solution of ynone 1 (0.2 $\mathrm{mmol})$ in DCM $(0.5 \mathrm{~mL})$ was added DMPU/HBr $(60 \mathrm{wt} / \mathrm{wt} \%)$ (54 $\mathrm{mg}, 0.4 \mathrm{mmol}$ ) at $0{ }^{\circ} \mathrm{C}$, then the mixture was warmed to room temperature and was stirred for $8 \mathrm{~h}$. After completion, the solvent was evaporated under the reduced pressure and the residue was purified by silica gel column chromatography (hexanes/EtOAc as elute solvent) to afford the corresponding products 3.

(Z)-4-Bromo-4-phenylbut-3-en-2-one (3a). ${ }^{23}$ Purified by flash column chromatography (ethyl acetate/hexanes $=1: 25$ ), pale yellow oil, $33.8 \mathrm{mg}, 76 \%$ yield. ${ }^{1} \mathrm{H}$ NMR (400 MHz, chloroform- $d$ ) $\delta 7.62$ (dd, $J=7.0,2.6 \mathrm{~Hz}, 2 \mathrm{H}), 7.45-7.29(\mathrm{~m}, 3 \mathrm{H}), 6.99(\mathrm{~s}, 1 \mathrm{H}), 2.43(\mathrm{~s}$, $3 \mathrm{H}) .{ }^{13} \mathrm{C}\left\{{ }^{1} \mathrm{H}\right\}$ NMR (100 MHz, chloroform- $\left.d\right) \delta 196.68,139.30$, 133.99, 130.44, 128.55, 128.26, 128.14, 127.90, 31.77.

(Z)-1-Bromo-4,4-dimethyl-1-phenylpent-1-en-3-one (3b). Purified by flash column chromatography (ethyl acetate/hexanes $=1: 25$ ), yellow oil, $43 \mathrm{mg}$, $81 \%$ yield. ${ }^{1} \mathrm{H}$ NMR ( $500 \mathrm{MHz}$, chloroform- $d$ ) $\delta$ 7.73-7.54 (m, 2H), 7.43-7.40 (m, 3H), $7.24(\mathrm{~s}, 1 \mathrm{H}), 1.24(\mathrm{~s}, 9 \mathrm{H})$. ${ }^{13} \mathrm{C}\left\{{ }^{1} \mathrm{H}\right\}$ NMR (126 MHz, chloroform- $d$ ) $\delta 204.10,139.83,134.18$, 130.16, 128.52, 128.10, 124.01, 44.38, 26.27. HRMS (ESI-FT-ICR) calcd. for $\mathrm{C}_{13} \mathrm{H}_{16} \mathrm{BrO}[\mathrm{M}+\mathrm{H}]^{+}: 267.0385$, found: 267.0378 .

(Z)-3-Bromo-1-cyclohexyl-3-phenylprop-2-en-1-one (3c). Purified by flash column chromatography (ethyl acetate/hexanes $=1: 25$ ), yellow oil, $46.1 \mathrm{mg}, 79 \%$ yield. ${ }^{1} \mathrm{H}$ NMR (500 MHz, chloroform- $d$ ) $\delta$ 7.74-7.57 (m, 2H), 7.52-7.30 (m, 3H), $7.07(\mathrm{~s}, 1 \mathrm{H}), 2.57-2.52(\mathrm{~m}$, $1 \mathrm{H}), 2.06-1.90(\mathrm{~m}, 2 \mathrm{H}), 1.85-1.82(\mathrm{~m}, 2 \mathrm{H}), 1.74-1.63(\mathrm{~m}, 1 \mathrm{H})$, $1.54-1.11(\mathrm{~m}, 5 \mathrm{H}) .{ }^{13} \mathrm{C}\left\{{ }^{1} \mathrm{H}\right\}$ NMR $(126 \mathrm{MHz}$, chloroform-d) $\delta 201.9$, 139.6, 133.6, 130.2, 128.5, 128.1, 126.4, 51.7, 28.3, 25.9, 25.6. HRMS (ESI-FT-ICR) calcd. for $\mathrm{C}_{15} \mathrm{H}_{18} \mathrm{BrO}[\mathrm{M}+\mathrm{H}]^{+}$: 293.0541, found: 293.0534.

(Z)-1-Bromo-1-phenyloct-1-en-3-one (3d). Purified by flash column chromatography (ethyl acetate/hexanes $=1: 25$ ), yellow oil, $44.8 \mathrm{mg}, 80 \%$ yield. ${ }^{1} \mathrm{H}$ NMR ( $500 \mathrm{MHz}$, chloroform- $d$ ) $\delta 7.80-7.54$ $(\mathrm{m}, 2 \mathrm{H}), 7.46-7.31(\mathrm{~m}, 3 \mathrm{H}), 7.01(\mathrm{~s}, 1 \mathrm{H}), 2.66(\mathrm{t}, J=7.4 \mathrm{~Hz}, 2 \mathrm{H})$, $1.70-1.68(\mathrm{~m}, 2 \mathrm{H}), 1.50-1.20(\mathrm{~m}, 4 \mathrm{H}), 0.92(\mathrm{t}, J=7.0 \mathrm{~Hz}, 3 \mathrm{H})$. ${ }^{13} \mathrm{C}\left\{{ }^{1} \mathrm{H}\right\}$ NMR (126 MHz, chloroform- $d$ ) $\delta 199.2,139.5,133.3,130.3$, 128.5, 128.1, 127.2, 44.4, 31.4, 23.6, 22.5, 13.9. HRMS (ESI-FT-ICR) calcd. for $\mathrm{C}_{14} \mathrm{H}_{18} \mathrm{BrO}[\mathrm{M}+\mathrm{H}]^{+}$: 281.0541, found:281.0535.

Methyl (Z)-3-(1-Bromo-3-oxooct-1-en-1-yl)benzoate (3e). Purified by flash column chromatography (ethyl acetate/hexanes $=1: 15$ ), yellow oil, $54.7 \mathrm{mg}, 81 \%$ yield. ${ }^{1} \mathrm{H}$ NMR ( $500 \mathrm{MHz}$, chloroform- $d$ ) $\delta$ $8.26(\mathrm{~s}, 1 \mathrm{H}), 8.06(\mathrm{~d}, J=8.2 \mathrm{~Hz}, 1 \mathrm{H}), 7.82(\mathrm{~d}, J=7.8 \mathrm{~Hz}, 1 \mathrm{H}), 7.48(\mathrm{t}$, $J=7.8 \mathrm{~Hz}, 1 \mathrm{H}), 7.05(\mathrm{~s}, 1 \mathrm{H}), 3.94(\mathrm{~s}, 3 \mathrm{H}), 2.66(\mathrm{t}, J=7.3 \mathrm{~Hz}, 2 \mathrm{H})$, $1.86-1.52(\mathrm{~m}, 2 \mathrm{H}), 1.34(\mathrm{dt}, J=7.7,3.6 \mathrm{~Hz}, 4 \mathrm{H}), 0.90(\mathrm{t}, J=6.8 \mathrm{~Hz}$, $3 \mathrm{H}) .{ }^{13} \mathrm{C}\left\{{ }^{1} \mathrm{H}\right\}$ NMR (126 MHz, chloroform-d) $\delta 199.0,166.2,139.8$, $132.5,131.7,131.1,130.6,128.9,128.8,128.0,52.4,44.4,31.3,23.5$, 22.5, 13.9. HRMS (ESI-FT-ICR) calcd. for $\mathrm{C}_{16} \mathrm{H}_{20} \mathrm{BrO}_{3}[\mathrm{M}+\mathrm{H}]^{+}$: 339.0596, found:339.0589.

(Z)-4-(4-Acetylphenyl)-4-bromobut-3-en-2-one (3f). Purified by flash column chromatography (ethyl acetate/hexanes $=1: 10$ ), yellow oil, $42.5 \mathrm{mg}$, 80\% yield. ${ }^{1} \mathrm{H}$ NMR (500 MHz, chloroform- $d$ ) $\delta 197.05$, 196.47, 143.34, 138.15, 131.97, 129.30, 128.45, 128.36, 31.66, 26.73. ${ }^{13} \mathrm{C}\left\{{ }^{1} \mathrm{H}\right\}$ NMR (126 MHz, chloroform-d) $\delta 197.1,196.5,143.3,138.2$, 132.0, 129.3, 128.5, 128.4, 31.7, 26.7. HRMS (ESI-FT-ICR) calcd. for $\mathrm{C}_{12} \mathrm{H}_{12} \mathrm{BrO}_{2}[\mathrm{M}+\mathrm{H}]^{+}:$267.0012, found: 267.0014 .

(Z)-4-Bromo-4-(4-bromophenyl)but-3-en-2-one (3g). Purified by flash column chromatography (ethyl acetate/hexanes $=1: 20$ ), yellow oil, $43.3 \mathrm{mg}$, $72 \%$ yield. ${ }^{1} \mathrm{H}$ NMR ( $500 \mathrm{MHz}$, chloroform- $d$ ) $\delta 7.54$ (d, $J=8.3 \mathrm{~Hz}, 2 \mathrm{H}), 7.50(\mathrm{~d}, J=8.7 \mathrm{~Hz}, 2 \mathrm{H}), 6.98(\mathrm{~s}, 1 \mathrm{H}), 2.44(\mathrm{~s}, 3 \mathrm{H})$. ${ }^{13} \mathrm{C}\left\{{ }^{1} \mathrm{H}\right\}$ NMR (126 MHz, chloroform- $d$ ) $\delta 196.4,138.2,132.4,131.8$, 129.6, 128.2, 124.9, 31.7. HRMS (ESI-FT-ICR) calcd. for $\mathrm{C}_{10} \mathrm{H}_{9} \mathrm{Br}_{2} \mathrm{O}$ $[\mathrm{M}+\mathrm{H}]^{+}:$302.9015, found:302.9013.

(Z)-4-Bromo-4-(3-methoxyphenyl)but-3-en-2-one (3h). Purified by flash column chromatography (ethyl acetate/hexanes $=1: 15$ ), yellow oil, $24.9 \mathrm{mg}$, $49 \%$ yield. ${ }^{1} \mathrm{H}$ NMR (500 MHz, chloroform- $d$ ) $\delta$
7.30 (d, $J=7.8 \mathrm{~Hz}, 2 \mathrm{H}), 7.21(\mathrm{~d}, J=7.7 \mathrm{~Hz}, 2 \mathrm{H}), 7.16(\mathrm{~s}, 1 \mathrm{H}), 7.00$ $(\mathrm{s}, 1 \mathrm{H}), 6.96(\mathrm{~d}, J=8.2 \mathrm{~Hz}, 2 \mathrm{H}), 3.85(\mathrm{~s}, 3 \mathrm{H}), 2.45(\mathrm{~s}, 3 \mathrm{H}) .{ }^{13} \mathrm{C}\left\{{ }^{1} \mathrm{H}\right\}$ NMR (126 MHz, chloroform-d) $\delta 196.7,159.5,140.7,133.6,129.5$, 128.1, 120.4, 116.0, 113.9, 55.4, 31.7. HRMS (ESI-FT-ICR) calcd. for $\mathrm{C}_{11} \mathrm{H}_{12} \mathrm{BrO}_{2}[\mathrm{M}+\mathrm{H}]^{+}:$255.0021, found: 255.0013 .

Methyl (Z)-3-(1-Bromo-3-oxobut-1-en-1-yl)benzoate (3i). Purified by flash column chromatography (ethyl acetate/hexanes $=1: 15$ ), yellow oil, $44.9 \mathrm{mg}$, $80 \%$ yield. ${ }^{1} \mathrm{H}$ NMR (500 MHz, chloroform- $d$ ) $\delta$ $8.27(\mathrm{~s}, 1 \mathrm{H}), 8.07(\mathrm{~d}, J=7.8 \mathrm{~Hz}, 1 \mathrm{H}), 7.83(\mathrm{~d}, J=7.5 \mathrm{~Hz}, 1 \mathrm{H}), 7.49(\mathrm{t}$, $J=7.8 \mathrm{~Hz}, 1 \mathrm{H}), 7.05(\mathrm{~s}, 1 \mathrm{H}), 3.95(\mathrm{~s}, 3 \mathrm{H}), 2.45(\mathrm{~s}, 3 \mathrm{H}) .{ }^{13} \mathrm{C}\left\{{ }^{1} \mathrm{H}\right\}$ NMR (126 MHz, chloroform-d) $\delta 196.4,166.2,139.7,132.5,132.4$, $131.3,130.6,129.0,128.8,128.7,52.4,31.7$. HRMS (ESI-FT-ICR) calcd. for $\mathrm{C}_{12} \mathrm{H}_{12} \mathrm{BrO}_{3}[\mathrm{M}+\mathrm{H}]^{+}$: 282.9970 , found: 282.9963 .

(Z)-3-Bromo-1-(4-nitrophenyl)-3-phenylprop-2-en-1-one (3j). Purified by flash column chromatography (ethyl acetate/hexanes $=1: 20$ ), yellow oil, $51.5 \mathrm{mg}, 78 \%$ yield. ${ }^{1} \mathrm{H}$ NMR ( $500 \mathrm{MHz}$, chloroform- $d$ ) $\delta$ $8.35(\mathrm{~d}, J=8.9 \mathrm{~Hz}, 2 \mathrm{H}), 8.16(\mathrm{~d}, J=8.9 \mathrm{~Hz}, 2 \mathrm{H}), 7.79-7.68(\mathrm{~m}, 2 \mathrm{H})$, $7.55(\mathrm{~s}, 1 \mathrm{H}), \quad 7.51-7.43(\mathrm{~m}, 3 \mathrm{H}) .{ }^{13} \mathrm{C}\left\{{ }^{1} \mathrm{H}\right\}$ NMR $(126 \mathrm{MHz}$, chloroform-d) $\delta 188.6,150.4,142.04,138.79,137.17,130.91,129.66$, $128.11,124.15,123.98$. HRMS (ESI-FT-ICR) calcd. for $\mathrm{C}_{15} \mathrm{H}_{11} \mathrm{BrNO}_{3}$ $[\mathrm{M}+\mathrm{H}]^{+}:$331.9922, found: 331.9914 .

Hydrobromination of Ynamides 4. To a solution of ynamides 4 $(0.2 \mathrm{mmol})$ in DCM $(0.5 \mathrm{~mL})$ was added DMPU/HBr $(60 \mathrm{wt} / \mathrm{wt} \%)$ (54 mg, $0.4 \mathrm{mmol}$ ) at $0{ }^{\circ} \mathrm{C}$, then the mixture was warmed to room temperature and was stirred for $8 \mathrm{~h}$. After completion, the solvent was evaporated under the reduced pressure and the residue was purified by silica gel column chromatography (hexanes/EtOAc as elute solvent) to afford the corresponding products $\mathbf{5}$.

(E)-N-Benzyl-N-(1-bromo-2-phenylvinyl)-4-methylbenzenesulfonamide (5a). ${ }^{1}$ Purified by flash column chromatography (ethyl acetate $/$ hexanes $=1: 20$ ), white solid, $72.4 \mathrm{mg}, 82 \%$ yield. Mp 142-143 ${ }^{\circ} \mathrm{C} .{ }^{1} \mathrm{H}$ NMR $(400 \mathrm{MHz}$, chloroform- $d$ ) $\delta 7.86(\mathrm{~d}, J=8.1 \mathrm{~Hz}, 2 \mathrm{H})$, $7.34(\mathrm{~d}, J=8.1 \mathrm{~Hz}, 2 \mathrm{H}), 7.33-7.27(\mathrm{~m}, 2 \mathrm{H}), 7.24(\mathrm{dd}, J=6.2,1.9 \mathrm{~Hz}$, $2 \mathrm{H}), 7.23-7.04(\mathrm{~m}, 6 \mathrm{H}), 6.81(\mathrm{~s}, 1 \mathrm{H}), 4.83(\mathrm{~d}, J=13.1 \mathrm{~Hz}, 1 \mathrm{H}), 3.94$ $(\mathrm{d}, J=13.1 \mathrm{~Hz}, 1 \mathrm{H}), 2.46(\mathrm{~s}, 3 \mathrm{H}) \cdot{ }^{13} \mathrm{C}\left\{{ }^{1} \mathrm{H}\right\} \operatorname{NMR}(100 \mathrm{MHz},) \delta$ $144.7,139.7,134.3,133.6,133.2,129.9,129.6,129.2$, 128.7, 128.6, 128.3, 128.1, 128.0, 119.6, 53.3, 21.7.

(E)-N-(1-Bromo-2-phenylvinyl)-N-heptyl-4-methylbenzenesulfonamide (5b). Purified by flash column chromatography (ethyl acetate/ hexanes $=1: 25)$, pale yellow oil, $85 \mathrm{mg}$, $86 \%$ yield. ${ }^{1} \mathrm{H}$ NMR (400 $\mathrm{MHz}$, chloroform- $d) \delta 7.82(\mathrm{~d}, J=8.2 \mathrm{~Hz}, 2 \mathrm{H}), 7.76-7.61(\mathrm{~m}, 2 \mathrm{H})$, 7.66-7.23 (m, 5H), $6.98(\mathrm{~s}, 1 \mathrm{H}), 3.62-3.59(\mathrm{~m}, 1 \mathrm{H}), 2.89-2.87(\mathrm{~m}$, $1 \mathrm{H}), 2.44(\mathrm{~s}, 3 \mathrm{H}), 1.77-1.35(\mathrm{~m}, 2 \mathrm{H}), 1.35-0.93(\mathrm{~m}, 8 \mathrm{H}), 0.82(\mathrm{t}, J=$ $7.0 \mathrm{~Hz}, 3 \mathrm{H}) .{ }^{13} \mathrm{C}\left\{{ }^{1} \mathrm{H}\right\} \mathrm{NMR}(100 \mathrm{MHz},) \delta 144.6,139.0,134.0,133.8$, 129.4, 129.2, 129.0, 120.2, 49.7, 31.6, 28.8, 27.4, 26.9, 22.5, 21.7, 14.0. HRMS (ESI-FT-ICR) calcd. for $\mathrm{C}_{22} \mathrm{H}_{29} \mathrm{BrNO}_{2} \mathrm{~S}[\mathrm{M}+\mathrm{H}]^{+}:$: 450.1102, found: 450.1099 .

(E)- $\mathrm{N}$-(1-Bromo-2-phenylvinyl)- $N$-methylmethanesulfonamide (5c). ${ }^{10}$ Purified by flash column chromatography (ethyl acetate/ hexanes $=1: 20$ ), pale yellow solid, $49 \mathrm{mg}, 85 \%$ yield. $\mathrm{Mp} 90-92{ }^{\circ} \mathrm{C}$. ${ }^{1} \mathrm{H}$ NMR (400 MHz, chloroform-d) $\delta 7.8-7.4(\mathrm{~m}, 2 \mathrm{H}), 7.36-7.31$ $(\mathrm{m}, 3 \mathrm{H}), 6.9(\mathrm{~s}, 1 \mathrm{H}), 3.1(\mathrm{~s}, 3 \mathrm{H}), 3.0(\mathrm{~s}, 3 \mathrm{H}) .{ }^{13} \mathrm{C}\left\{{ }^{1} \mathrm{H}\right\}$ NMR $(100$ $\mathrm{MHz}$ ) $\delta 137.3,133.4,129.2,128.8,128.6,120.5,37.4,36.5 . \mathrm{HRMS}$ (ESI-FT-ICR) calcd. for $\mathrm{C}_{10} \mathrm{H}_{13} \mathrm{BrNO}_{2} \mathrm{~S}[\mathrm{M}+\mathrm{H}]^{+}:$289.9850, found: 289.9842 .

(E)-N-(1-Bromo-2-phenylvinyl)- $N$-phenylmethanesulfonamide (5d). ${ }^{10}$ White solid, $58 \mathrm{mg}, 83 \%$ yield. Mp $136-137{ }^{\circ} \mathrm{C} .{ }^{1} \mathrm{H}$ NMR $(400 \mathrm{MHz}$, chloroform- $d) \delta 8.03-7.36(\mathrm{~m}, 4 \mathrm{H}), 7.54-7.14(\mathrm{~m}, 6 \mathrm{H})$, $7.06(\mathrm{~s}, 1 \mathrm{H}), 3.14(\mathrm{~s}, 3 \mathrm{H}) .{ }^{13} \mathrm{C}\left\{{ }^{1} \mathrm{H}\right\} \mathrm{NMR}(100 \mathrm{MHz},) \delta 138.9,138.5$, 133.3, 128.7, 128.6, 127.4, 123.7, 117.9, 39.0. HRMS (ESI-FT-ICR) calcd. for $\mathrm{C}_{15} \mathrm{H}_{15} \mathrm{BrNO}_{2} \mathrm{~S}[\mathrm{M}+\mathrm{H}]^{+}$: 352.0007 , found: 352.0001 .

(E)-N-(1-Bromo-2-(2-chlorophenyl)vinyl)-N,4-dimethylbenzenesulfonamide (5e). Purified by flash column chromatography (ethyl acetate $/$ hexanes $=1: 20$ ), yellow solid, $62 \mathrm{mg}, 78 \%$ yield. $\mathrm{Mp} 82-83{ }^{\circ} \mathrm{C}$. ${ }^{1} \mathrm{H}$ NMR $(400 \mathrm{MHz}$, chloroform- $d$ ) $\delta 7.80(\mathrm{dd}, J=6.7,2.8 \mathrm{~Hz}, 1 \mathrm{H})$, $7.71(\mathrm{dd}, J=8.4,1.6 \mathrm{~Hz}, 2 \mathrm{H}), 7.43-7.29(\mathrm{~m}, 1 \mathrm{H}), 7.26(\mathrm{dd}, J=7.2$, $3.4 \mathrm{~Hz}, 4 \mathrm{H}), 7.17(\mathrm{~s}, 1 \mathrm{H}), 2.95(\mathrm{~s}, 3 \mathrm{H}), 2.42(\mathrm{~s}, 3 \mathrm{H}) .{ }^{13} \mathrm{C}\left\{{ }^{1} \mathrm{H}\right\} \mathrm{NMR}$ $(100 \mathrm{MHz},) \delta 144.6,133.5,133.4,133.3,132.3,129.6,129.5,126.9$, 123.5, 36.9, 21.6. HRMS $\left(\mathrm{ESI}^{+}\right.$) calcd. for $\mathrm{C}_{16} \mathrm{H}_{16} \mathrm{BrClNO}_{2} \mathrm{~S}[\mathrm{M}+\mathrm{H}]^{+}$: 399.9774, found: 399.9770 . 
(E)-N-(1-Bromo-2-(3-chlorophenyl)vinyl)-N,4-dimethylbenzenesulfonamide (5f). Purified by flash column chromatography (ethyl acetate/hexanes $=1: 20)$, white solid, $65.2 \mathrm{mg}$, $82 \%$ yield. Mp 113-114 ${ }^{\circ} \mathrm{C}$. ${ }^{1} \mathrm{H}$ NMR (400 MHz, chloroform- $d$ ) $\delta 7.83-7.67(\mathrm{~m}, 2 \mathrm{H}), 7.50(\mathrm{~s}$, $1 \mathrm{H}), 7.46(\mathrm{~s}, 1 \mathrm{H}), 7.28(\mathrm{t}, J=6.5 \mathrm{~Hz}, 4 \mathrm{H}), 6.79(\mathrm{~d}, J=2.0 \mathrm{~Hz}, 1 \mathrm{H})$, $2.98(\mathrm{~s}, 3 \mathrm{H}), 2.43(\mathrm{~s}, 3 \mathrm{H}) .{ }^{13} \mathrm{C}\left\{{ }^{1} \mathrm{H}\right\}$ NMR $(100 \mathrm{MHz},) \delta 144.6,133.5$, 133.4, 133.5, 132.3, 129.6, 129.5, 126.9, 123.5, 36.9, 21.6. HRMS (ESIFT-ICR) calcd. for $\mathrm{C}_{16} \mathrm{H}_{16} \mathrm{BrClNO}_{2} \mathrm{~S}[\mathrm{M}+\mathrm{H}]^{+}:$399.9774, found: 399.9766.

(E)-N-(1-Bromo-2-(4-chlorophenyl)vinyl)-N,4-dimethylbenzenesulfonamide $(5 \mathrm{~g})$. Purified by flash column chromatography (ethyl acetate/hexanes $=1: 20$ ), yellow solid, $62.8 \mathrm{mg}$, 79\% yield. Mp $142-$ $143{ }^{\circ} \mathrm{C} .{ }^{1} \mathrm{H}$ NMR $(400 \mathrm{MHz}$, chloroform-d) $\delta 7.76(\mathrm{~d}, J=8.3 \mathrm{~Hz}$, $2 \mathrm{H}), 7.52(\mathrm{~d}, J=8.6 \mathrm{~Hz}, 2 \mathrm{H}), 7.33-7.27(\mathrm{~m}, 4 \mathrm{H}), 6.59(\mathrm{~s}, 1 \mathrm{H}), 3.02$ (s, 3H), $2.44(\mathrm{~s}, 3 \mathrm{H}) .{ }^{13} \mathrm{C}\left\{{ }^{1} \mathrm{H}\right\} \mathrm{NMR}(100 \mathrm{MHz},) \delta 144.6,134.5,134.1$, 131.6, 131.1, 129.9, 129.5, 128.9, 128.7, 35.7, 21.6. HRMS (ESI-FTICR) calcd. for $\mathrm{C}_{16} \mathrm{H}_{16} \mathrm{BrClNO}_{2} \mathrm{~S}[\mathrm{M}+\mathrm{H}]^{+}$: 399.9774, found: 399.9764.

(E)-N-(1-Bromo-2-(4-fluorophenyl)vinyl)- $N$,4-dimethylbenzenesulfonamide (5h). Purified by flash column chromatography (ethyl acetate $/$ hexanes $=1: 20)$, white solid, $62.6 \mathrm{mg}$, $82 \%$ yield. Mp $86-88$ ${ }^{\circ} \mathrm{C} .{ }^{1} \mathrm{H}$ NMR $(400 \mathrm{MHz}$, chloroform- $d) \delta 7.77(\mathrm{~d}, J=7.7 \mathrm{~Hz}, 2 \mathrm{H})$, $7.67-7.51(\mathrm{~m}, 2 \mathrm{H}), 7.30(\mathrm{~d}, J=7.9 \mathrm{~Hz}, 2 \mathrm{H}), 7.03(\mathrm{t}, J=8.3 \mathrm{~Hz}, 2 \mathrm{H})$, $6.81(\mathrm{~s}, 1 \mathrm{H}), 2.97(\mathrm{~s}, 3 \mathrm{H}), 2.43(\mathrm{~s}, 3 \mathrm{H}) .{ }^{19} \mathrm{~F}$ NMR $(376 \mathrm{MHz}$, chloroform- $d) \delta-111.2(\mathrm{dq}, J=9.0,5.0,4.3 \mathrm{~Hz}) .{ }^{13} \mathrm{C}\left\{{ }^{1} \mathrm{H}\right\} \operatorname{NMR}(100$ $\mathrm{MHz}$, chloroform- $d$ ) $\delta 162.7(\mathrm{~d}, J=250.0 \mathrm{~Hz}), 144.7,135.8,133.5$, 130.7, 130.6, 129.5, 129.0, 121.1, 115.7 (d, $J=21.7 \mathrm{~Hz}), 36.6$, 21.6. HRMS (ESI-FT-ICR) calcd. for $\mathrm{C}_{16} \mathrm{H}_{16} \mathrm{BrFNO}_{2} \mathrm{~S}[\mathrm{M}+\mathrm{H}]^{+}$: 384.0069, found: 384.0061 .

(E)-N-(1-Bromo-2-(4-bromophenyl)vinyl)-N-phenylmethanesulfonamide (5i). Purified by flash column chromatography (ethyl acetate/ hexanes $=1: 20$ ), white solid, $69 \mathrm{mg}, 81 \%$ yield. $\mathrm{Mp} 121-122{ }^{\circ} \mathrm{C} .{ }^{1} \mathrm{H}$ NMR (400 MHz, chloroform- $d$ ) $\delta 7.53(\mathrm{~d}, J=8.1 \mathrm{~Hz}, 2 \mathrm{H}), 7.44(\mathrm{q}, J$ $=8.4 \mathrm{~Hz}, 4 \mathrm{H}), 7.35(\mathrm{t}, J=7.6 \mathrm{~Hz}, 2 \mathrm{H}), 7.29(\mathrm{~d}, J=6.6 \mathrm{~Hz}, 2 \mathrm{H}), 6.99$ (s, 1H), $3.14(\mathrm{~s}, 3 \mathrm{H}) .{ }^{13} \mathrm{C}\left\{{ }^{1} \mathrm{H}\right\} \mathrm{NMR}(100 \mathrm{MHz},) \delta 138.7,137.3,132.2$, 131.9, 130.1, 129.6, 127.6, 123.6, 123.3, 118.6, 39.0. HRMS (ESI-FTICR) calcd. for $\mathrm{C}_{15} \mathrm{H}_{14} \mathrm{Br}_{2} \mathrm{NO}_{2} \mathrm{~S}[\mathrm{M}+\mathrm{H}]^{+}: 429.9107$, found: 429.9100 .

(E)-N-(1-Bromo-2-(4-cyanophenyl)vinyl)- $N$,4-dimethylbenzenesulfonamide (5j). Purified by flash column chromatography (ethyl acetate/hexanes $=1: 15)$, colorless oil, $64 \mathrm{mg}, 82 \%$ yield. Mp 131-134 ${ }^{\circ} \mathrm{C} .{ }^{1} \mathrm{H}$ NMR $(400 \mathrm{MHz}$, chloroform- $d) \delta \delta 7.75(\mathrm{~d}, J=7.7 \mathrm{~Hz}, 2 \mathrm{H})$, $7.68(\mathrm{~d}, J=8.0 \mathrm{~Hz}, 2 \mathrm{H}), 7.62(\mathrm{~d}, J=7.9 \mathrm{~Hz}, 2 \mathrm{H}), 7.31(\mathrm{~d}, J=7.8 \mathrm{~Hz}$, $2 \mathrm{H}), 6.87(\mathrm{~s}, 1 \mathrm{H}), 2.98(\mathrm{~s}, 3 \mathrm{H}), 2.44(\mathrm{~s}, 3 \mathrm{H}) .{ }^{13} \mathrm{C}\left\{{ }^{1} \mathrm{H}\right\}$ NMR $(100$ $\mathrm{MHz}$,) $\delta 145.1,138.2,135.1,133.1,132.4,129.6,129.1,129.0,124.9$, 118.6, 112.0, 36.8, 21.7. HRMS (ESI-FT-ICR) calcd. for $\mathrm{C}_{17} \mathrm{H}_{16} \mathrm{BrN}_{2} \mathrm{O}_{2} \mathrm{~S}[\mathrm{M}+\mathrm{H}]^{+}: 391.0116$, found: 391.0106 .

Methyl (E)-3-(2-Bromo-2-(N-phenylmethylsulfonamido)vinyl) benzoate (5k). Purified by flash column chromatography (ethyl acetate/hexanes $=1: 15$ ), colorless oil, $52.4 \mathrm{mg}, 64 \%$ yield. ${ }^{1} \mathrm{H}$ NMR $(400 \mathrm{MHz}$, chloroform- $d) \delta 8.15(\mathrm{~s}, 1 \mathrm{H}), 7.97(\mathrm{~d}, J=6.8 \mathrm{~Hz}, 1 \mathrm{H})$, $7.85(\mathrm{~d}, J=8.1 \mathrm{~Hz}, 1 \mathrm{H}), 7.57(\mathrm{~d}, J=8.1 \mathrm{~Hz}, 2 \mathrm{H}), 7.43(\mathrm{t}, J=7.8 \mathrm{~Hz}$, $1 \mathrm{H}), 7.34(\mathrm{t}, J=7.6 \mathrm{~Hz}, 2 \mathrm{H}), 7.28(\mathrm{~d}, J=7.1 \mathrm{~Hz}, 1 \mathrm{H}), 7.08(\mathrm{~s}, 1 \mathrm{H})$, $3.90(\mathrm{~s}, 3 \mathrm{H}), 3.13(\mathrm{~s}, 3 \mathrm{H}) .{ }^{13} \mathrm{C}\left\{{ }^{1} \mathrm{H}\right\}$ NMR $(100 \mathrm{MHz},) \delta 166.5,138.9$, 137.2 , 133.6, 132.5, 130.5, 130.0, 129.9, 129.6, 128.9, 127.7, 124.0, 119.3, 52.2, 39.0. HRMS (ESI-FT-ICR) calcd. for $\mathrm{C}_{17} \mathrm{H}_{17} \mathrm{BrNO}_{4} \mathrm{~S}[\mathrm{M}$ $+\mathrm{H}]^{+}$: 410.0062, found: 410.0054 .

Hydrochlorination of Ynamides 4. To a solution of ynamides 4 $(0.2 \mathrm{mmol})$ in DCM $(0.5 \mathrm{~mL})$ was added DMPU/HCl $(43 \mathrm{wt} / \mathrm{wt} \%)$ (34 mg, $0.4 \mathrm{mmol}$ ) at $0{ }^{\circ} \mathrm{C}$, then the mixture was warmed to room temperature and was stirred for $8 \mathrm{~h}$. After completion, the solvent was evaporated under the reduced pressure and the residue was purified by silica gel column chromatography (hexanes/EtOAc as elute solvent) to afford the corresponding products 6 .

(E)-N-(1-Chloro-2-phenylvinyl)- $N$-methylmethanesulfonamide (6a). ${ }^{10}$ Purified by flash column chromatography (ethyl acetate/ hexanes = 1:15), white solid, $46.5 \mathrm{mg}$, 95\% yield. ${ }^{1} \mathrm{H}$ NMR $(400 \mathrm{MHz}$, chloroform-d) $\delta 7.56(\mathrm{~d}, J=7.0 \mathrm{~Hz}, 2 \mathrm{H}), 7.34-7.30(\mathrm{~m}, 3 \mathrm{H}), 6.68(\mathrm{~s}$, $1 \mathrm{H}), 3.13(\mathrm{~s}, 3 \mathrm{H}), 3.00(\mathrm{~s}, 3 \mathrm{H}) .{ }^{13} \mathrm{C}\left\{{ }^{1} \mathrm{H}\right\}$ NMR $(100 \mathrm{MHz},) \delta 132.7$, 132.5, 129.1, 128.8, 128.6, 38.1, 35.7. HRMS (ESI-FT-ICR) calcd. for $\mathrm{C}_{10} \mathrm{H}_{13} \mathrm{ClNO}_{2} \mathrm{~S}[\mathrm{M}+\mathrm{H}]^{+}:$246.0356, found: 246.0350 .
(E)-N-(1-Chloro-2-phenylvinyl)-N-heptyl-4-methylbenzenesulfonamide (6b). Purified by flash column chromatography (ethyl acetate/ hexanes $=1: 20)$, colorless oil, $72.9 \mathrm{mg}, 85 \%$ yield. ${ }^{1} \mathrm{H}$ NMR $(400$ $\mathrm{MHz}$, chloroform- $d$ ) $\delta 7.81(\mathrm{~d}, J=8.3 \mathrm{~Hz}, 2 \mathrm{H}), 7.70(\mathrm{~d}, J=7.1 \mathrm{~Hz}$, $2 \mathrm{H}), 7.34-7.30(\mathrm{~m}, 5 \mathrm{H}), 6.77(\mathrm{~s}, 1 \mathrm{H}), 3.56(\mathrm{~s}, 1 \mathrm{H}), 3.01(\mathrm{~s}, 1 \mathrm{H}), 2.43$ (s, 3H), 1.53 (bs, 2H), 1.55-0.94 (m, $8 \mathrm{H}), 0.82(\mathrm{t}, J=7.0 \mathrm{~Hz}, 3 \mathrm{H})$.. ${ }^{13} \mathrm{C}\left\{{ }^{1} \mathrm{H}\right\}$ NMR $(100 \mathrm{MHz},) \delta 144.4,134.7,134.4,133.1,129.5,129.1$, 129.0, 128.9, 128.5, 127.9, 48.6, 31.6, 28.8, 27.3, 26.8, 22.4, 21.6, 14.0. HRMS (ESI-FT-ICR) calcd. for $\mathrm{C}_{22} \mathrm{H}_{28} \mathrm{ClNO}_{2} \mathrm{~S}[\mathrm{M}+\mathrm{H}]^{+}$: 406.1608, found: 406.1599 .

(E)-N-Benzyl-N-(1-chloro-2-phenylvinyl)-4-methylbenzenesulfonamide (6c). Purified by flash column chromatography (ethyl acetate/ hexanes $=1: 20)$, white solid, $74.6 \mathrm{mg}, 94 \%$ yield. $\mathrm{Mp} 127-129^{\circ} \mathrm{C} .{ }^{1} \mathrm{H}$ NMR (400 MHz, chloroform- $d$ ) $\delta 7.86(\mathrm{~d}, J=8.3 \mathrm{~Hz}, 2 \mathrm{H}), 7.42-7.28$ $(\mathrm{m}, 4 \mathrm{H}), 7.24-7.00(\mathrm{~m}, 8 \mathrm{H}), 6.60(\mathrm{~s}, 1 \mathrm{H}), 4.80(\mathrm{~d}, J=12.3 \mathrm{~Hz}, 1 \mathrm{H})$, $4.04(\mathrm{~d}, J=12.3 \mathrm{~Hz}, 1 \mathrm{H}), 2.46(\mathrm{~s}, 3 \mathrm{H}) .{ }^{13} \mathrm{C}\left\{{ }^{1} \mathrm{H}\right\} \operatorname{NMR}(100 \mathrm{MHz},) \delta$ 144.6, 135.0, 134.9, 133.3, 132.8, 129.8, 129.6, 128.9, 128.7, 128.5, 128.3, 128.2, 128.0, 127.5, 52.4, 21.7. HRMS (ESI-FT-ICR) calcd. for $\mathrm{C}_{22} \mathrm{H}_{21} \mathrm{ClNO}_{2} \mathrm{~S}[\mathrm{M}+\mathrm{H}]^{+}:$398.0982, found: 398.0980 .

(E)-N-(1-Chloro-2-phenylvinyl)- $N$-phenylmethanesulfonamide (6d). ${ }^{10}$ Purified by flash column chromatography (ethyl acetate/ hexanes $=1: 15)$, pale yellow liquid, $60.1 \mathrm{mg}$, $95 \%$ yield. ${ }^{1} \mathrm{H}$ NMR (400 $\mathrm{MHz}$, chloroform- $d) \delta 7.83-7.41(\mathrm{~m}, 4 \mathrm{H}), 7.46-7.07(\mathrm{~m}, 6 \mathrm{H}), 6.84$ $(\mathrm{s}, 1 \mathrm{H}), 3.13(\mathrm{~s}, 3 \mathrm{H}) .{ }^{13} \mathrm{C}\left\{{ }^{1} \mathrm{H}\right\} \mathrm{NMR}(100 \mathrm{MHz},) \delta 138.8,133.6,132.6$, 129.5, 129.1, 128.7, 128.6, 128.1, 127.6, 124.3, 39.6. HRMS (ESI-FTICR) calcd. for $\mathrm{C}_{15} \mathrm{H}_{15} \mathrm{ClNO}_{2} \mathrm{~S}[\mathrm{M}+\mathrm{H}]^{+}:$: 308.0512, found: 308.0506 .

(E)-N-Allyl-N-(1-chloro-2-phenylvinyl)-4-methylbenzenesulfonamide (6e). ${ }^{10}$ Purified by flash column chromatography (ethyl acetate/ hexanes $=1: 20)$, yellow solid, $66 \mathrm{mg}, 95 \%$ yield. ${ }^{1} \mathrm{H}$ NMR $(400 \mathrm{MHz}$, chloroform- $d$ ) $\delta 7.81(\mathrm{~d}, J=8.3 \mathrm{~Hz}, 2 \mathrm{H}), 7.67(\mathrm{~d}, J=7.1 \mathrm{~Hz}, 2 \mathrm{H})$, 7.50-7.18 (m, 5H), $6.74(\mathrm{~s}, 1 \mathrm{H}), 5.72-5.71(\mathrm{~m}, 1 \mathrm{H}), 5.24-5.23(\mathrm{~m}$, $1 \mathrm{H}), 5.12-5.10(\mathrm{~m}, 1 \mathrm{H}), 4.28(\mathrm{~s}, 1 \mathrm{H}), 3.64(\mathrm{~s}, 1 \mathrm{H}), 2.44(\mathrm{~s}, 3 \mathrm{H})$. ${ }^{13} \mathrm{C}\left\{{ }^{1} \mathrm{H}\right\}$ NMR (100 MHz, $\delta 144.5,134.7,134.4,133.2,130.5,129.5$, 129.0, 128.7, 128.8, 128.5, 127.6, 120.9, 51.5, 21.6. HRMS (ESI-FTICR) calcd. for $\mathrm{C}_{18} \mathrm{H}_{19} \mathrm{ClNO}_{2} \mathrm{~S}[\mathrm{M}+\mathrm{H}]^{+}: 348.0825$, found: 348.0817 .

(E)- $N$-(1-Chloro-2-phenylvinyl)- $N$-(2-(cyclohex-1-en-1-yl)ethyl)-4methylbenzenesulfonamide (6f). Purified by flash column chromatography (ethyl acetate $/$ hexanes $=1: 25$ ), yellow oil, $68 \mathrm{mg}, 82 \%$ yield. ${ }^{1} \mathrm{H}$ NMR (400 MHz, chloroform-d) $\delta 7.81(\mathrm{~d}, J=8.3 \mathrm{~Hz}, 2 \mathrm{H}), 7.69$ (d, $J=7.4 \mathrm{~Hz}, 2 \mathrm{H}), 7.43-7.26(\mathrm{~m}, 5 \mathrm{H}), 6.76(\mathrm{~s}, 1 \mathrm{H}), 5.33(\mathrm{~s}, 1 \mathrm{H})$, $3.71(\mathrm{~s}, 1 \mathrm{H}), 3.05(\mathrm{~s}, 1 \mathrm{H}), 2.43(\mathrm{~s}, 3 \mathrm{H}), 2.18-2.15(\mathrm{~m}, 2 \mathrm{H}), 1.86-$ $1.83(\mathrm{~m}, 4 \mathrm{H}), 1.52-1.49(\mathrm{~m}, 4 \mathrm{H}) .{ }^{13} \mathrm{C}\left\{{ }^{1} \mathrm{H}\right\}$ NMR $(100 \mathrm{MHz},) \delta$ $144.4,134.7,134.5,133.4,133.1,129.5,129.3,128.8,128.4,127.6$, 123.9, 47.1, 35.6, 28.1, 25.1, 22.7, 22.1, 21.6. HRMS (ESI ${ }^{+}$) calcd. for $\mathrm{C}_{23} \mathrm{H}_{27} \mathrm{ClNO}_{2} \mathrm{~S}[\mathrm{M}+\mathrm{H}]^{+}: 416.1446$, found: 416.1441 .

(E)-3-(1-Chloro-2-phenylvinyl)oxazolidin-2-one (6g). Purified by flash column chromatography (ethyl acetate/hexanes $=1: 10$ ), white solid, $41 \mathrm{mg}$, 92\% yield. Mp $124-126{ }^{\circ} \mathrm{C} .{ }^{1} \mathrm{H}$ NMR $(400 \mathrm{MHz}$, chloroform-d) $7.31(\mathrm{~s}, 5 \mathrm{H}), 6.73(\mathrm{~s}, 1 \mathrm{H}), 4.42(\mathrm{t}, J=7.8 \mathrm{~Hz}, 2 \mathrm{H}), 3.76$ $(\mathrm{t}, J=7.9 \mathrm{~Hz}, 2 \mathrm{H}) .{ }^{13} \mathrm{C}\left\{{ }^{1} \mathrm{H}\right\} \mathrm{NMR}(100 \mathrm{MHz},) \delta 155.3,132.9,130.4$, $128.8,128.7,127.9,125.9,62.8,44.5$. HRMS (ESI-FT-ICR) calcd. for $\mathrm{C}_{11} \mathrm{H}_{11} \mathrm{ClNO}_{2}[\mathrm{M}+\mathrm{H}]^{+}: 224.0448$, found: 224.0441 .

(E)-N-(1-Chloro-2-(naphthalen-1-yl)vinyl)- $N$-methylmethanesulfonamide (6h). Purified by flash column chromatography (ethyl acetate/hexanes $=1: 20$ ), pale yellow liquid, $53 \mathrm{mg}, 90 \%$ yield. ${ }^{1} \mathrm{H}$ NMR (400 MHz, chloroform- $d$ ) $\delta 7.94(\mathrm{~d}, J=7.6 \mathrm{~Hz}, 1 \mathrm{H}), 7.84(\mathrm{t}, J=$ $9.2 \mathrm{~Hz}, 2 \mathrm{H}), 7.76(\mathrm{~d}, J=7.2 \mathrm{~Hz}, 1 \mathrm{H}), 7.62-7.43(\mathrm{~m}, 3 \mathrm{H}), 7.32(\mathrm{~s}$, $1 \mathrm{H}), 3.02(\mathrm{~s}, 3 \mathrm{H}), 2.81(\mathrm{~s}, 3 \mathrm{H}) .{ }^{13} \mathrm{C}\left\{{ }^{1} \mathrm{H}\right\} \operatorname{NMR}(100 \mathrm{MHz},) \delta 133.4$, $131.5,131.2,130.1,130.0,129.1,128.7,126.6,126.5,126.1,125.6$, $123.8,38.5$, 36.3. HRMS (ESI-FT-ICR) calcd. for $\mathrm{C}_{14} \mathrm{H}_{15} \mathrm{ClNO}_{2} \mathrm{~S}[\mathrm{M}$ $+\mathrm{H}]^{+}: 296.0512$, found: 296.0506 .

(E)-N-(1-Chloro-2-(4-fluorophenyl)vinyl)-N,4-dimethylbenzenesulfonamide (6i). ${ }^{24}$ Purified by flash column chromatography (ethyl acetate $/$ hexanes $=1: 20)$, pale yellow solid, $63.7 \mathrm{mg}, 94 \%$ yield. ${ }^{1} \mathrm{H}$ NMR (400 MHz, chloroform-d) $\delta 7.77(\mathrm{~d}, J=8.1 \mathrm{~Hz}, 2 \mathrm{H}), 7.64-7.54$ $(\mathrm{m}, 2 \mathrm{H}), 7.30(\mathrm{~d}, J=7.9 \mathrm{~Hz}, 2 \mathrm{H}), 7.09-6.99(\mathrm{~m}, 2 \mathrm{H}), 6.60(\mathrm{~s}, 1 \mathrm{H})$, $3.01(\mathrm{~s}, 3 \mathrm{H}), 2.43$ (s, 3H). ${ }^{19} \mathrm{~F}$ NMR $(376 \mathrm{MHz}$, chloroform- $d) \delta$ $-111.5--111.6(\mathrm{~m}) .{ }^{13} \mathrm{C}\left\{{ }^{1} \mathrm{H}\right\}$ NMR (100 MHz, chloroform- $\left.d\right) \delta$ $162.7(\mathrm{~d}, J=249.7 \mathrm{~Hz}), 144.6,134.1,131.1,130.6(\mathrm{~d}, J=8.3 \mathrm{~Hz})$, $129.5,129.3,128.8,115.7(\mathrm{~d}, J=21.7 \mathrm{~Hz}), 35.7,21.6$. 
(E)-N-(1-Chloro-2-(4-chlorophenyl)vinyl)-N-4-dimethylbenzenesulfonamide (6j). Purified by flash column chromatography (ethyl acetate/hexanes $=1: 20)$, white solid, $61.7 \mathrm{mg}, 87 \%$ yield. Mp 108-110 ${ }^{\circ} \mathrm{C} .{ }^{1} \mathrm{H}$ NMR (400 MHz, chloroform- $\left.d\right) \delta 7.76(\mathrm{~d}, J=7.9 \mathrm{~Hz}, 2 \mathrm{H})$, $7.52(\mathrm{~d}, J=7.5 \mathrm{~Hz}, 2 \mathrm{H}), 7.30(\mathrm{~d}, J=7.6 \mathrm{~Hz}, 4 \mathrm{H}), 6.80(\mathrm{~s}, 1 \mathrm{H}), 2.97$ (s, 3H), 2.44 (s, 3H). ${ }^{13} \mathrm{C}\left\{{ }^{1} \mathrm{H}\right\}$ NMR (100 MHz,) $\delta$ 144.8, 135.7, 134.6 , 133.5, 132.3, 129.9, 129.5, 129.0, 128.9, 122.0, 36.7, 21.6. HRMS (ESI-FT-ICR) calcd. for $\mathrm{C}_{16} \mathrm{H}_{16} \mathrm{Cl}_{2} \mathrm{NO}_{2} \mathrm{~S}[\mathrm{M}+\mathrm{H}]^{+}$: 356.0279, found:356.0272.

(E)-N-(1-Chloro-2-(4-methoxyphenyl)vinyl)- $N$-methylmethanesulfonamide (6k). Purified by flash column chromatography (ethyl acetate/hexanes = 1:15), white solid, $50.6 \mathrm{mg}$, 92\% yield. Mp 125-127 ${ }^{\circ} \mathrm{C}{ }^{1} \mathrm{H}$ NMR (500 MHz, chloroform- $d$ ) $\delta 7.54(\mathrm{~d}, J=8.7 \mathrm{~Hz}, 2 \mathrm{H})$, $6.89(\mathrm{~d}, J=8.7 \mathrm{~Hz}, 2 \mathrm{H}), 6.62(\mathrm{~s}, 1 \mathrm{H}), 3.81(\mathrm{~s}, 3 \mathrm{H}), 3.14(\mathrm{~s}, 3 \mathrm{H}), 3.03$ $(\mathrm{s}, 3 \mathrm{H}) .{ }^{13} \mathrm{C}\left\{{ }^{1} \mathrm{H}\right\}$ NMR $(125 \mathrm{MHz}$ ) $\delta 160.1,132.0,130.2,126.9,125.4$, 114.1, 55.3, 38.0, 35.6. HRMS (ESI-FT-ICR) calcd. for $\mathrm{C}_{11} \mathrm{H}_{15} \mathrm{ClNO}_{3} \mathrm{~S}$ $[\mathrm{M}+\mathrm{H}]^{+}: 276.0461$, found:276.0453.

(E)-N-(1-Chloro-2-(4-nitrophenyl)vinyl)-N-4-dimethylbenzenesulfonamide (6l). Purified by flash column chromatography (ethyl acetate $/$ hexanes $=1: 15)$, white solid, $64.4 \mathrm{mg}$, $88 \%$ yield. Mp 154-157 ${ }^{\circ} \mathrm{C} .{ }^{1} \mathrm{H}$ NMR (500 MHz, chloroform-d) $\delta 8.21(\mathrm{~d}, J=8.9 \mathrm{~Hz}, 2 \mathrm{H})$, 7.77 (d, $J=7.5 \mathrm{~Hz}, 4 \mathrm{H}), 7.33$ (d, $J=8.4 \mathrm{~Hz}, 2 \mathrm{H}), 3.06(\mathrm{~s}, 3 \mathrm{H}), 2.46$ (s, 3H). ${ }^{13} \mathrm{C}\left\{{ }^{1} \mathrm{H}\right\}$ NMR $(126 \mathrm{MHz},) \delta 147.3,145.0,139.6,133.7$, 133.6, 130.1, 129.7, 129.4, 128.7, 123.9, 35.8, 21.6. HRMS (ESI-FTICR) calcd. for $\mathrm{C}_{16} \mathrm{H}_{16} \mathrm{ClN}_{2} \mathrm{O}_{4} \mathrm{~S}[\mathrm{M}+\mathrm{H}]^{+}: 367.0519$, found 367.0512 .

(E)-N-(2-(4-Acetylphenyl)-1-chlorovinyl)-N-4-dimethylbenzenesulfonamide $(6 \mathrm{~m})$. Purified by flash column chromatography (ethyl acetate/hexanes = 1:10), white solid, $66 \mathrm{mg}, 91 \%$ yield. Mp 142-143 ${ }^{\circ} \mathrm{C} .{ }^{1} \mathrm{H}$ NMR $(400 \mathrm{MHz}$, chloroform- $d$ ) $\delta \delta 7.93(\mathrm{~d}, J=8.4 \mathrm{~Hz}, 2 \mathrm{H})$, $7.75(\mathrm{~d}, J=8.1 \mathrm{~Hz}, 2 \mathrm{H}), 7.67(\mathrm{~d}, J=7.9 \mathrm{~Hz}, 2 \mathrm{H}), 7.30(\mathrm{~d}, J=7.8 \mathrm{~Hz}$, $2 \mathrm{H}), 6.68(\mathrm{~s}, 1 \mathrm{H}), 3.03(\mathrm{~s}, 3 \mathrm{H}), 2.59(\mathrm{~s}, 3 \mathrm{H}), 2.42(\mathrm{~s}, 3 \mathrm{H}) .{ }^{13} \mathrm{C}\left\{{ }^{1} \mathrm{H}\right\}$ NMR $(100 \mathrm{MHz}) \delta 197.3,144.7,137.7,136.7,133.9,132.1,131.2$, $129.6,128.8,128.7,128.6,35.6,26.6,21.6$. HRMS (ESI-FT-ICR) calcd. for $\mathrm{C}_{18} \mathrm{H}_{19} \mathrm{ClNO}_{3} \mathrm{~S}[\mathrm{M}+\mathrm{H}]^{+}: 364.0774$, found: 364.0766 .

(E)-N-(1-Chloro-2-(thiophen-3-yl)vinyl)-N,4-dimethylbenzenesulfonamide (6n). Purified by flash column chromatography (ethyl acetate/hexanes $=1: 15$ ), white solid, $58.8 \mathrm{mg}$, 90\% yield. Mp 139-141 ${ }^{\circ} \mathrm{C}$. ${ }^{1} \mathrm{H}$ NMR $(500 \mathrm{MHz}$, chloroform- $d) \delta 7.84(\mathrm{~d}, J=6.8 \mathrm{~Hz}, 2 \mathrm{H})$, $7.38(\mathrm{~d}, J=4.4 \mathrm{~Hz}, 1 \mathrm{H}), 7.34(\mathrm{~d}, J=7.7 \mathrm{~Hz}, 2 \mathrm{H}), 7.15(\mathrm{~d}, J=3.5 \mathrm{~Hz}$, $1 \mathrm{H}), 7.00(\mathrm{t}, J=3.9 \mathrm{~Hz}, 1 \mathrm{H}), 6.88(\mathrm{~s}, 1 \mathrm{H}), 3.05(\mathrm{~s}, 3 \mathrm{H}), 2.46(\mathrm{~s}, 3 \mathrm{H})$. ${ }^{13} \mathrm{C}\left\{{ }^{1} \mathrm{H}\right\}$ NMR $(126 \mathrm{MHz},) \delta 144.6,135.9,134.1,129.9,129.5,129.1$, $128.3,127.0,126.7,126.4,35.5,21.7$. HRMS (ESI-FT-ICR) calcd. for $\mathrm{C}_{14} \mathrm{H}_{15} \mathrm{ClNO}_{2} \mathrm{~S}_{2}[\mathrm{M}+\mathrm{H}]^{+}: 328.0233$, found: 328.0225 .

(3S, 10R, 13R, 17R)-10,13-Dimethyl-17-((R)-6-methylheptan-2-yl)2,3,4,7,8,9,10,11,12,13,14,15,16,17-tetradecahydro-1H-cyclopenta[a]phenanthren-3-yl $\quad(E)-6$-chloro-6-( $(N, 4$-dimethylphenyl)sulfonamido)hex-5-enoate (60). Purified by flash column chromatography (ethyl acetate/hexanes = 1:20), white solid, $114 \mathrm{mg}, 82 \%$ yield. Mp 88-89 ${ }^{\circ} \mathrm{C} .{ }^{1} \mathrm{H}$ NMR (500 MHz, chloroform- $d \delta 7.76(\mathrm{~d}, J=$ $8.2 \mathrm{~Hz}, 2 \mathrm{H}), 7.32(\mathrm{~d}, J=8.0 \mathrm{~Hz}, 2 \mathrm{H}), 5.79(\mathrm{t}, J=7.5 \mathrm{~Hz}, 1 \mathrm{H}), 5.38(\mathrm{~d}$, $J=5.1 \mathrm{~Hz}, 1 \mathrm{H}), 4.64(\mathrm{dq}, J=11.9,7.0,5.6 \mathrm{~Hz}, 1 \mathrm{H}), 2.92(\mathrm{~s}, 3 \mathrm{H}), 2.44$ $(\mathrm{s}, 3 \mathrm{H}), 2.36-2.32(\mathrm{~m}, 6 \mathrm{H}), 2.05-1.93(\mathrm{~m}, 2 \mathrm{H}), 1.92-1.79(\mathrm{~m}, 3 \mathrm{H})$, $1.68-1.06(\mathrm{~m}, 23 \mathrm{H}), 1.03(\mathrm{~s}, 3 \mathrm{H}), 0.92(\mathrm{~d}, J=6.5 \mathrm{~Hz}, 3 \mathrm{H}), 0.88(\mathrm{~d}, J$ $=2.3 \mathrm{~Hz}, 3 \mathrm{H}), 0.87(\mathrm{~d}, J=2.3 \mathrm{~Hz}, 3 \mathrm{H}), 0.68(\mathrm{~s}, 3 \mathrm{H}) \cdot{ }^{13} \mathrm{C}\left\{{ }^{1} \mathrm{H}\right\} \mathrm{NMR}$ $(126 \mathrm{MHz}$ ) $\delta 172.6,144.3,139.7,134.3,134.0,129.5,129.0,128.5$, 122.6, 74.0, 56.7, 56.1, 50.0, 42.3, 39.7, 39.5, 38.1, 37.0, 36.6, 36.2, $35.9,35.8,34.1,31.9,31.9,28.6,28.2,28.0,27.8,24.3,23.9,23.8,22.8$, 22.6, 21.6, 21.0, 19.3, 18.7. HRMS (ESI-FT-ICR) calcd. for $\mathrm{C}_{41} \mathrm{H}_{63} \mathrm{ClNO}_{4} \mathrm{~S}[\mathrm{M}+\mathrm{H}]^{+}: 700.4166$, found: 700.4158.

Further Transformations of Halogenated Alkene Products. A mixture of $2 \mathrm{a}(0.2 \mathrm{mmol})$, phenylboronic acid $(36.5 \mathrm{mg}, 0.3 \mathrm{mmol})$, $\mathrm{Pd}\left(\mathrm{PPh}_{3}\right)_{2} \mathrm{Cl}_{2}(7 \mathrm{mg}, 5 \mathrm{~mol} \%)$, and $\mathrm{Cs}_{2} \mathrm{CO}_{3}(652 \mathrm{mg}, 2 \mathrm{mmol})$ was added 1,4-dioxane $/ \mathrm{H}_{2} \mathrm{O}(4 / 1,2 \mathrm{~mL})$ heated at $60{ }^{\circ} \mathrm{C}$ for $8 \mathrm{~h}$. After the completion of the reaction, the reaction mixture extracted with EtOAc, the combined organic layers dried over $\mathrm{Na}_{2} \mathrm{SO}_{4}$ and removal of the solvent by rotary evaporation the resulting residue was purified by chromatography on silica gel to give the desired products.

4,4-Diphenylbut-3-en-2-one $(7 a){ }^{25}{ }^{1} \mathrm{H}$ NMR $(400 \mathrm{MHz}$, chloroform- $d) \delta 7.50-7.36(\mathrm{~m}, 3 \mathrm{H}), 7.39-7.27(\mathrm{~m}, 5 \mathrm{H}), 7.23-7.18(\mathrm{~m}$, $1 \mathrm{H}), 6.58(\mathrm{~s}, 1 \mathrm{H}), 1.88(\mathrm{~s}, 3 \mathrm{H}) \cdot{ }^{13} \mathrm{C}\left\{{ }^{1} \mathrm{H}\right\}$ NMR $(100 \mathrm{MHz}$, chloroform- $d) \delta 200.2, \quad 154.0, \quad 140.8, \quad 138.9,129.6,129.5,128.8$, $128.4,128.4,127.7,30.3$.

A mixture of $4 \mathrm{a}(73.5 \mathrm{mg}, 0.3 \mathrm{mmol})$, phenylboronic acid $(51.2 \mathrm{mg}$, $4.2 \mathrm{mmol}), \mathrm{Pd}(\mathrm{OAc})_{2}(7 \mathrm{mg}, 10 \mathrm{~mol} \%)$, and $\mathrm{K}_{2} \mathrm{CO}_{3}(82.8 \mathrm{mg}, 0.6$ $\mathrm{mmol})$ in $\mathrm{EtOH} / \mathrm{H}_{2} \mathrm{O}(4 / 1)(2 \mathrm{~mL})$ was heated at $80{ }^{\circ} \mathrm{C}$ for $8 \mathrm{~h}$. After the completion of the reaction, the reaction mixture was filtered through a pad of Celite and removal of the solvent in vacuum to give the crude product. The resulting residue was purified by silica gel chromatography to give the desired products.

(Z)-N-(1,2-Diphenylvinyl)-N-methylmethanesulfonamide (Z-8a). Purified by flash column chromatography (ethyl acetate/hexanes = 1:40), colorless oil, $40.2 \mathrm{mg}, 47 \%$ yield. ${ }^{1} \mathrm{H}$ NMR $(500 \mathrm{MHz}$, chloroform- $d) \delta 7.57(\mathrm{~d}, J=8.8 \mathrm{~Hz}, 2 \mathrm{H}), 7.52(\mathrm{~d}, J=7.2 \mathrm{~Hz}, 2 \mathrm{H})$, $7.45-7.41(\mathrm{~m}, 5 \mathrm{H}), 7.33(\mathrm{~d}, J=7.4 \mathrm{~Hz}, 1 \mathrm{H}), 6.83(\mathrm{~s}, 1 \mathrm{H}), 3.12(\mathrm{~s}$, $3 \mathrm{H}), 2.50(\mathrm{~s}, 3 \mathrm{H}) .{ }^{13} \mathrm{C}\left\{{ }^{1} \mathrm{H}\right\} \mathrm{NMR}(126 \mathrm{MHz},) \delta 139.6,137.7,135.7$, 128.9, 128.8, 128.7, 128.7, 128.1, 127.0, 40.0, 37.1. HRMS (ESI-FTICR) calcd. for $\mathrm{C}_{16} \mathrm{H}_{17} \mathrm{NO}_{2} \mathrm{~S}[\mathrm{M}+\mathrm{H}]^{+}: 288.1058$, found: 288.1050 .

(E)-N-(1,2-Diphenylvinyl)- $N$-methylmethanesulfonamide (E-8a). Purified by flash column chromatography (ethyl acetate/hexanes = 1:40), white solid, $37.2 \mathrm{mg}$, $43 \%$ yield. Mp $113-115{ }^{\circ} \mathrm{C}{ }^{1} \mathrm{H}$ NMR $(500 \mathrm{MHz}$, chloroform-d) $\delta 7.37$ (dd, $J=7.1,2.8 \mathrm{~Hz}, 2 \mathrm{H}), 7.32(\mathrm{dd}, J$ $=5.2,1.9 \mathrm{~Hz}, 3 \mathrm{H}), 7.16-7.12(\mathrm{~m}, 3 \mathrm{H}), 7.06-6.98(\mathrm{~m}, 2 \mathrm{H}), 6.75(\mathrm{~s}$, $1 \mathrm{H}), 3.13(\mathrm{~s}, 3 \mathrm{H}), 2.99(\mathrm{~s}, 3 \mathrm{H}) .{ }^{13} \mathrm{C}\left\{{ }^{1} \mathrm{H}\right\} \operatorname{NMR}(125 \mathrm{MHz},) \delta 140.0$, 135.4, 135.2, 129.8, 129.2, 128.8, 128.7, 128.1, 127.5, 127.3, 39.2, 37.1. HRMS (ESI-FT-ICR) calcd. for $\mathrm{C}_{16} \mathrm{H}_{18} \mathrm{NO}_{2} \mathrm{~S}[\mathrm{M}+\mathrm{H}]^{+}: 288.1058$, found: 288.1051 .

A mixture of $5 \mathrm{c}(87 \mathrm{mg}, 0.3 \mathrm{mmol})$, ethynyltrimethylsilane $(41.2$ $\mathrm{mg}, 4.2 \mathrm{mmol}), \mathrm{Pd}\left(\mathrm{PPh}_{3}\right) \mathrm{Cl}_{2}(10 \mathrm{mg}, 5 \mathrm{~mol} \%)$, and $\mathrm{CuI}(5 \mathrm{mg}, 8 \mathrm{~mol}$ $\%)$ in $\mathrm{Et}_{3} \mathrm{~N} / \mathrm{THF}(1 / 1 ; 1.5 \mathrm{~mL})$ was stirred at room temperature for 8 h. After the completion of the reaction, the reaction mixture was filtered through Celite and removal of the solvent in vacuum gave the crude product. The crude product was purified by silica gel chromatography (ethyl acetate/hexanes $=1: 30$ ) to give the desired product $9 \mathrm{a}$ as a pale-yellow oil ( $87 \mathrm{mg}, 95 \%$ yield). Major isomer: ${ }^{1} \mathrm{H}$ NMR $(400 \mathrm{MHz}$, chloroform- $d$ ) $\delta 7.84(\mathrm{dd}, J=7.6,2.0 \mathrm{~Hz}, 2 \mathrm{H})$, $7.42-7.27(\mathrm{~m}, 3 \mathrm{H}), 7.05(\mathrm{~s}, 1 \mathrm{H}), 3.12(\mathrm{~s}, 3 \mathrm{H}), 2.94(\mathrm{~s}, 3 \mathrm{H}), 0.26(\mathrm{~s}$, $9 \mathrm{H}) .{ }^{13} \mathrm{C}\left\{{ }^{1} \mathrm{H}\right\}$ NMR $(125 \mathrm{MHz},) \delta 140.3,133.5,129.5,129.3,128.3$, 118.4, 104.3, 99.0, 35.7, -0.4. Minor isomer: ${ }^{1} \mathrm{H}$ NMR $(400 \mathrm{MHz}$, chloroform-d) $\delta 7.70(\mathrm{~d}, J=8.1 \mathrm{~Hz}, 2 \mathrm{H}), 6.80(\mathrm{~s}, 1 \mathrm{H}), 3.05(\mathrm{~s}, 3 \mathrm{H})$, $3.01(\mathrm{~s}, 3 \mathrm{H}), 0.23(\mathrm{~s}, 8 \mathrm{H}) .{ }^{13} \mathrm{C}\left\{{ }^{1} \mathrm{H}\right\}$ NMR $(126 \mathrm{MHz},) \delta 139.0,133.6$, 129.9, 128.7, 119.9, 101.0, 97.1, 36.4, -0.3. HRMS (ESI-FT-ICR) calcd. for $\mathrm{C}_{15} \mathrm{H}_{22} \mathrm{NO}_{2} \mathrm{SSi}[\mathrm{M}+\mathrm{H}]^{+}$: 308.1141, found: 308.1133 .

\section{ASSOCIATED CONTENT}

\section{S Supporting Information}

The Supporting Information is available free of charge on the ACS Publications website at DOI: 10.1021/acs.joc.7b02257.

$$
\text { Copies of NMR spectra (PDF) }
$$

\section{AUTHOR INFORMATION}

\section{Corresponding Authors}

*E-mail: gb.hammond@louisville.edu

*E-mail: bo.xu@dhu.edu.cn

\section{ORCID}

Gerald B. Hammond: 0000-0002-9814-5536

Notes

The authors declare no competing financial interest.

\section{ACKNOWLEDGMENTS}

We are grateful to the National Science Foundation of China for financial support (NSFC-21672035) and China Recruitment Program of Global Experts for financial support. G.B.H. is grateful to the National Institutes of Health (R01GM121660). X.Z is grateful to the China Scholarship Council for financial support. 


\section{REFERENCES}

(1) (a) Johansson Seechurn, C. C. C.; Kitching, M. O.; Colacot, T. J.; Snieckus, V. Angew. Chem., Int. Ed. 2012, 51, 5062-5085. (b) Iwai, T.; Fujihara, T.; Terao, J.; Tsuji, Y. J. Am. Chem. Soc. 2009, 131, 66686669. (c) Petrone, D. A.; Franzoni, I.; Ye, J.; Rodríguez, J. F.; Poblador-Bahamonde, A. I.; Lautens, M. J. Am. Chem. Soc. 2017, 139, 3546-3557. (d) Uehling, M. R.; Rucker, R. P.; Lalic, G. J. Am. Chem. Soc. 2014, 136, 8799-8803. (e) Yamaguchi, R.; Kawasaki, H.; Kawanisi, M. Synth. Commun. 1982, 12, 1027-1037. (f) Zhu, G.; Chen, D.; Wang, Y.; Zheng, R. Chem. Commun. 2012, 48, 5796-5798. (g) Dérien, S.; Klein, H.; Bruneau, C. Angew. Chem., Int. Ed. 2015, 54, 12112-12115.

(2) (a) Baran, P. S.; Shenvi, R. A.; Mitsos, C. A. Angew. Chem., Int. Ed. 2005, 44, 3714-3717. (b) Chevolot, L.; Chevolot, A. M.; Gajhede, M.; Larsen, C.; Anthoni, U.; Christophersen, C. J. Am. Chem. Soc. 1985, 107, 4542-4543. (c) Sun, C.; Camp, J. E.; Weinreb, S. M. Org. Lett. 2006, 8, 1779-1781.

(3) (a) Weiss, H. M. J. Chem. Educ. 1995, 72, 848. (b) Podhajsky, S. M.; Sigman, M. S. Organometallics 2007, 26, 5680-5686. (c) Xu, C.X.; Ma, C.-H.; Xiao, F.-R.; Chen, H.-W.; Dai, B. Chin. Chem. Lett. 2016, 27, 1683-1685. (d) Oliver-Meseguer, J.; Doménech-Carbó, A.; Boronat, M.; Leyva-Pérez, A.; Corma, A. Angew. Chem., Int. Ed. 2017, 56, 6435-6439. (e) Wang, B.; Wang, S.; Li, P.; Wang, L. Chem. Commun. 2010, 46, 5891-5893. (f) Iwai, T.; Fujihara, T.; Terao, J.; Tsuji, Y. J. Am. Chem. Soc. 2012, 134, 1268-1274. (g) Schevenels, F. T.; Shen, M.; Snyder, S. A. J. Am. Chem. Soc. 2017, 139, 6329-6337.

(4) (a) Chelpanova, L. F.; Mashlyakovskii, L. N. Zh. Obshch. Khim. 1966, 2, 602-607. (b) Kerr, P. J.; Pyke, S. M.; Ward, A. D. Aust. J. Chem. 2008, 61, 350-358. (c) Shchukin, A. O.; Vasilyev, A. V. Appl. Catal., A 2008, 336, 140-147. (d) Semenova, I. S.; Yarovenko, V. N.; Levchenko, K. S.; Krayushkin, M. M. Russ. Chem. Bull. 2013, 62, 1022-1025. (e) Yan, W.; Li, Z.; Kishi, Y. J. Am. Chem. Soc. 2015, 137, $6219-6225$.

(5) (a) Kundu, N. G.; Choudhuri, L. N.; Das, B. J. Chem. Soc., Perkin Trans. 1 1989, 1, 1165-6. (b) Kundu, N. G.; Chaudhuri, L. N. J. Chem. Soc., Perkin Trans. 1 1991, 1, 1677-82.

(6) Yang, F.; Ji, K.-G.; Ali, S.; Liang, Y.-M. J. Org. Chem. 2011, 76, 8329-8335.

(7) Mulder, J. A.; Kurtz, K. C. M.; Hsung, R. P.; Coverdale, H.; Frederick, M. O.; Shen, L.; Zificsak, C. A. Org. Lett. 2003, 5, 15471550.

(8) (a) Ohashi, K.; Mihara, S.; Sato, A. H.; Ide, M.; Iwasawa, T. Tetrahedron Lett. 2014, 55, 632-635. (b) Sato, A. H.; Ohashi, K.; Iwasawa, T. Tetrahedron Lett. 2013, 54, 1309-1311.

(9) Prabagar, B.; Nayak, S.; Mallick, R. K.; Prasad, R.; Sahoo, A. K. Org. Chem. Front. 2016, 3, 110-115.

(10) Kim, S. W.; Um, T.-W.; Shin, S. Chem. Commun. 2017, 53, $2733-2736$.

(11) (a) Compain, G.; Jouvin, K.; Martin-Mingot, A.; Evano, G.; Marrot, J.; Thibaudeau, S. Chem. Commun. 2012, 48, 5196-5198. (b) Métayer, B.; Compain, G.; Jouvin, K.; Martin-Mingot, A.; Bachmann, C.; Marrot, J.; Evano, G.; Thibaudeau, S. J. Org. Chem. 2015, 80, 3397-3410.

(12) Zeng, X.; Liu, S.; Shi, Z.; Xu, B. Org. Lett. 2016, 18, 4770-4773. (13) (a) Okoromoba, O. E.; Hammond, G. B.; Xu, B. Org. Lett. 2015, 17, 3975-3977. (b) Okoromoba, O. E.; Han, J.; Hammond, G. B.; Xu, B. J. Am. Chem. Soc. 2014, 136, 14381-14384. (c) Okoromoba, O. E.; Li, Z.; Robertson, N.; Mashuta, M. S.; Couto, U. R.; Tormena, C. F.; $\mathrm{Xu}$, B.; Hammond, G. B. Chem. Commun. 2016, 52, 13353-13356.

(14) Laurence, C.; Brameld, K. A.; Graton, J.; Le Questel, J.-Y.; Renault, E. J. Med. Chem. 2009, 52, 4073-4086.

(15) (a) Liang, S.; Ebule, R.; Hammond, G. B.; Xu, B. Org. Lett. 2017, 19, 4524-4527. (b) Li, Z.; Ebule, R.; Kostyo, J.; Hammond, G. B.; Xu, B. Chem. - Eur. J. 2017, 23, 12739-12743.

(16) (a) DeKorver, K. A.; Li, H.; Lohse, A. G.; Hayashi, R.; Lu, Z.; Zhang, Y.; Hsung, R. P. Chem. Rev. 2010, 110, 5064-5106. (b) Evano, G.; Coste, A.; Jouvin, K. Angew. Chem., Int. Ed. 2010, 49, 2840-2859. (c) Wang, X.-N.; Yeom, H.-S.; Fang, L.-C.; He, S.; Ma, Z.-X.; Kedrowski, B. L.; Hsung, R. P. Acc. Chem. Res. 2014, 47, 560-578. (d) Hu, L.; Xu, S.; Zhao, Z.; Yang, Y.; Peng, Z.; Yang, M.; Wang, C.; Zhao, J. J. Am. Chem. Soc. 2016, 138, 13135-13138. (e) Xu, S.; Liu, J.; Hu, D.; Bi, X. Green Chem. 2015, 17, 184-187. (f) Zhang, Y. Tetrahedron 2006, 62, 3917-3927. (g) Wu, W.; Jiang, H. Acc. Chem. Res. 2014, 47, 2483-2504. (h) Li, J.; Yang, W.; Yang, S.; Huang, L.; Wu, W.; Sun, Y.; Jiang, H. Angew. Chem., Int. Ed. 2014, 53, 72197222. (i) Li, Y.; Liu, X.; Jiang, H.; Liu, B.; Chen, Z.; Zhou, P. Angew. Chem., Int. Ed. 2011, 50, 6341-6345. (j) Li, J.; Yang, S.; Huang, L.; Chen, H.; Jiang, H. RSC Adv. 2013, 3, 11529-11532.

(17) (a) Stang, P. J.; Summerville, R. J. Am. Chem. Soc. 1969, 91, 4600-1. (b) Zhang, S.-L.; Wan, H.-X.; Deng, Z.-Q. Org. Biomol. Chem. 2017, 15, 6367-6374.

(18) (a) Carey, F. A.; Sundberg, R. J. Advanced Organic Chemistry, Part A: Structure and Mechanisms. In Advanced Organic Chemistry, Part A: Structure and Mechanisms, 5th ed.; Springer, 2007; Vol. A, pp 536-540. (b) Ashtekar, K. D.; Vetticatt, M.; Yousefi, R.; Jackson, J. E.; Borhan, B. J. Am. Chem. Soc. 2016, 138, 8114-8119.

(19) (a) Weiss, H. M.; Touchette, K. M.; Andersen, F.; Iskhakov, D. Org. Biomol. Chem. 2003, 1, 2148. (b) Weiss, H. M.; Touchette, K. M.; Angell, S.; Khan, J. Org. Biomol. Chem. 2003, 1, 2152. (c) Weiss, H. M.; Touchette, K. M. J. Chem. Soc., Perkin Trans. 2 1998, 2, 1523-1528.

(20) Zeng, X.; Lu, Z.; Liu, S.; Hammond, G. B.; Xu, B. Adv. Synth. Catal.2017359406210.1002/adsc.201701179

(21) Doyle, M. P.; Devia, A. H.; Bassett, K. E.; Terpstra, J. W.; Mahapatro, S. N. J. Org. Chem. 1987, 52, 1619-1621.

(22) Hosseini-Sarvari, M.; Mardaneh, Z. Bull. Chem. Soc. Jpn. 2011, $84,778-782$.

(23) Shchukin, A. O.; Vasilyev, A. V. Appl. Catal., A 2008, 336, 140147.

(24) Prabagar, B.; Nayak, S.; Mallick, R. K.; Prasad, R.; Sahoo, A. K. Org. Chem. Front. 2016, 3, 110-115.

(25) Tani, M.; Sakaguchi, S.; Ishii, Y. J. Org. Chem. 2004, 69, 12211226. 